\title{
AN OVERVIEW OF COLLECTIVE EFFECTS IN CIRCULAR AND LINEAR ACCELERATORS ${ }^{\star}$
}

\author{
RONALD D. RUTH \\ Stanford Linear Accelerator Center, \\ Stanford University, Stanford, CA 94309
}

\begin{abstract}
DISCLAIMER
This report was prepared as an account of work sponsored by an agency of the United States Government. Neither the United States Government nor any agency thereof, nor any of their employets, makes any wartanty. express or implied, or assumes any legal liability or responsibility for the accuracy. completeness, or usefulsess of any information, apparatus, product, or process disclosen, or represents that its use would not infringe privately owned rights, Refer. ence herein to any specific commercial produer, process, or service by trade name, trademart, manufactuter, of otherwise does not necessarily constitute or imply its endorsement, recommendation, or favoring by the Uniled States Government or any agency thereof. The views and opinions of authors expressed herein do not necessarily state or reflect those of the United States Coverament or any agency thereot.
\end{abstract}

Invited talk presented at the 3rd Joint US-CERN School on Particle Accelerators: Frontiers of Particle Beeams, Observation, Diagnosis, and Correction, Capri, Italy, October 20-26, 1988

* Work supported by the Department of Energy, contract DE-AC03-76SF00515. 


\section{TABLE OF CONTENTS}

1 Introduction

2 Longitudinal Basics

2.1 The Equations of Motion

2.2 The Collective Force

2.2.1 The Longitudinal Wake Potential

2.2.2 The Impedance

2.3 Potential Well Distortion

3 Transverse Basics

3.1 Equations of Motion

3.2 The Collective Force

3.3 Potential Well Distortion

4 Longitudinal Effects

4.1 Linear Accelerators

4.1.1 Single Bunch

4.1.2 Multi-bunch

4.2 Circular Accelerators

4.2.1 Single Bunch
A. Coasting Beams
B. Fast Blow-up of Long Bunches
C. Mode Coupling

4.2.2 Multi-bunch

A. Symmetric Coupled Bunch Instabilities

5 Transverse Effects

5.1 Single Bunch

5.1.1 Linear Acceleratorg

A. Beam Break-up and BNS Damping

B. BNS Damping at the SLC

5.1.2 Circular Accelerators

A. Mode Coupling

B. The Head-Tail Effect

C. Fast Blow-up of Single Bunches

5.2 Multi-bunch

5.2.1 Linear Accelerators
A. Cumulative Beam Break-up
B. Cures for Beam Break-up

5.2.2 Circular Accelerators

A. Coupled Batches of Bunches

6 Concluding Pemarks and Acknowledgements

7 References 


\section{INTRODUCTION}

The purpose of both linear and circular accelerators is, of course, to accelerate beams of charged particles. In order to do this it is necessary not only to accelerate particles but also to confine them transversely so that they remain in the vacuum environment. Originally, as accelerators were developed, the intensity of the heams was rather low and so the external fields could be applied without regard to the effects of the space-charge forces of the beams. However, as the demand for high intensity increased, collective effects that are due to the space-charge forces became increasingly important.

What do we mean by collective effects? In order to control a beam of particles we apply external fields. These focus the beam transversely and accelerate it and focus it longitudinally. In addition to these externally applied fields a particle within the beam feels a field due to the charge and current of all the other particles in the beam. By collective effects, we mean all those modifications to the beam behavior which are due to these beam-induced forces. As an example, let's consider a beam of particles of one charge. If these particles are at nonrelativistic energies then they tend to repel one another. Thus, we need external focusing to control the natural divergence of the beam, and also to counteract the divergence due to space-charge forces. At higher energies, when $v \simeq c$, since like currents attract, the electric repulsion due to the charge of the beam and the magnet attraction due to the current in the beam tend to cancel. Unfortunately, the vacuum environment (RF cavities, bellows, beam position monitors, and other discontinuities) tends to spoil this cancellation of electric and magnetic effects. For this reason it's necessary to study the effect of these beam induced fields even at very high energy.

Since in this particular paper we are going to discuss many different effects, it's useful to discuss the philosophy of organization. Basically, we have a $2 \times 2 \times 2$ organizational problem. The first two major topics are linear and circular accelerators. In the linear accelerator case, we will consider as examples only electron linacs that have relatively high energy and so particles will have $v \simeq c$. For circular accelerators we'll consider both protons and electrons or their anti-particles.

The next two topics are single bunches and multi-bunches. In both linear accelerators and circular accelerators the particles have a bunched character because they are accelerated by an RF system, and the RF system has a natural waveiength. There is a particular point on the 
RF wave that's optimal for the bunch to sit. There are other non-optimal points that typically don't have any particles, and thus there is a bunched character to the beam. In many cases there is only a single bunch in an accelerator but in other cases there are many bunches. By single bunch effects, we mean those effects which happen within a single bunch. These are dominated by wakefields which tend to be strong over one bunch length. Multi-bunches are typically separated by a distance which is long compared to the size of the bunch and are coupled via a long-range wakefield. For this reason, we will tend to divide the problem into single bunches with rather short wakefields and multi-bunches with rather long wakefields. In the impedance domain, this means that we consider rather broad band impedances for single bunches and narrow band impedances for multi-bunches. There is an intermediate wakefield region which wjll also be discussed in the final section.

The next two topics arise from the natural separation of longitudinal and transverse effects. The two transverse degrees of freedom in an accelerator are rather similar and thus can be treated in the same way. This is not true for the longitudinal degree of freedom in both linear accelerators and circular accelerators. In an electron linac where the velocity is near the velocity of light, the particles have a fixed position relative to one another within the bunch. On the other hand, in a circular accelerator, for both electrons and protons, the particles of higher energy or lower energy travel different path lengths and have different velacities; thus they exchange their positions longitudinally. Therefore, the main difference between linear and circular accelerators is the circulation longitudinally in circular accelerators. The case of proton linacs is analogous to the case of circular accelerators; since the velocity is significantly less than the velocity of light, there is longitudinal circulation.

Since this paper is an overview of an extremely tich and broad field, it is impossible to cover all the material on all the subjects just listed. Therefore, in each case a few subtopics will be selected to illustrate the physics. There is a small amount of theory in the paper; in many cases the theoretical results are stated and sometimes data are given from experiments. From the overlap of subjects just discussed, we have 8 topics. These are collected in chapters 4 and 5 . In the next two chapters, we discuss the basics of longitudinal and transverse motion in storage rings. In these cases we consider the most straightforward of collective effects, potential well tistortion induced by the collective force of the distribution of particles in the beam. For more detailed articles and references, the reader is referred to the proceedings of U.S. and CERN Particle Accelerator Schools Jisted in Refs. 1-3. 


\section{LONGITUDINAL BASICS}

\subsection{The Equations of Motion}

In order to begin the discussion of longitudinal motion, it useful first to define the coordinate system. In Fig. 1, we see the coordinate system which will be used in the discussion of circular accelerators where

$$
\begin{aligned}
& s=\beta c t, \theta=\omega_{0} t, \text { revolution period } T_{0}=\frac{2 \pi}{\omega_{0}}, \\
& E_{0}=\text { reference energy }
\end{aligned}
$$

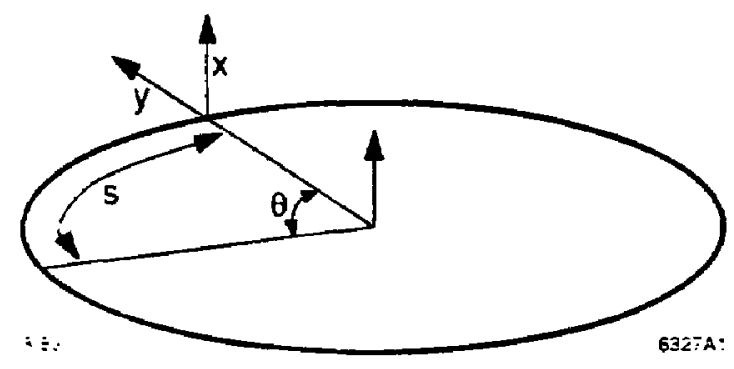

i iz. T. Tie courdinale systen for a circular accelerator.

It is useful to define beam coordinates. To do this we pick a reference particle rotating at $\omega_{0}$ and perfurm a transformation to a rotating coordinate system. That is, we let

$$
\theta=\phi+\omega_{0} t
$$

Thus, $\phi$ is just the angle reiative to the moving reference particle. In addition, it is useful to define the relativa energy deviation $\epsilon$

$$
\epsilon=\frac{E-E_{0}}{E_{0}}
$$

In many cases we use the longitudinal distance in terms of a time unit rather than an angular unit. In this case, we define the variable $\tau$ given by

$$
r=0 ; w_{i+1}^{\prime}
$$

$\tau$ is simply the "distance" from the reference particle in time units; it is sometimes more convenient : han the tariable $\phi$. 
Now let us derive the longitudinal equations of motion. If we consider tlie time derivative of $\phi$, we see that it is equal to the frequency deviation from the reference particle. That is

$$
\frac{d \phi}{d t}=\frac{d \theta}{d t}-\omega_{0}=\omega-\omega_{0}
$$

However, we know that the relative frequency difference in a beam is related to the momentum difference,

$$
\frac{\omega-\omega_{0}}{\omega_{0}}=\frac{\Delta \omega}{\omega_{0}} \simeq-\eta \frac{E-E_{0}}{E_{0}}=-\eta \epsilon
$$

where $\eta$ is the frequency slip factor. In terms of the momentum compaction factor $\alpha$ we have

$$
\eta=\alpha-\frac{1}{\gamma^{2}}
$$

Rewriting Eq. (2.5), we obtain

$$
\frac{d \phi}{d t}=-\eta \omega_{0} \epsilon
$$

Next we need an equation for the relative energy deviation $\epsilon$. The energy of the beam can change due to three effects. First, it changes due to the applied RF voltage. Next it can change due to the interaction of the beam with the environment. Finally, it changes due to synchrotron radiation of single particles in the bending field.

In this section, we will neglect the loss of energy due to synchrotron radiation although this is easy to include; it results in the shift of the stable phase angle. For the purposes of deriving a differential equation for $\epsilon$, let us assume that the change in energy is small in one turn. This allows us to express the diffe:ence in $\epsilon$ which is obtained in one turn as a differential with respect to time rather than a difference with respect to time. In this case, the energy gain is simply the integrated voltage gain in one turn; to obtain the equation for the derivative of $\epsilon$, we simply divide by the revolution time, which yields,

$$
\frac{d \epsilon}{d t}=\frac{e V(\phi, t)}{E_{0} T_{0}} .
$$

In this case $V(\phi, t)$ is the integrated voltage for one turn for a particle at $\phi$ and $t$ from all sources. The differential equation is a rather good approximation provided that $V$ is sufficiently small. Combining Eqs. (2.8) and (2.9) we obtain

$$
\frac{d^{2} \phi}{d t^{2}}=\frac{-2 \pi \eta e V(\phi, t)}{E_{0} T_{0}^{2}}
$$

If we rewrite Ec. (2.10) in terms of the variable $\tau$, we obtain

$$
\frac{d^{2} \tau}{d t^{2}}=-\frac{\eta e V(\tau, t)}{E_{0} T_{0}}
$$

Eq. (2.11) is a second-order differential equation for the longitudinal deviation from the reference particle. We will find that it is useful to construct a Hamiltonian for the motion. The 
effective Hamiltonian for Eq. (2.11) is given by

$$
H=\frac{p^{2}}{2}+\frac{\eta e}{E_{0} T_{0}} \int^{r} V\left(\tau^{\prime}, t\right) d \tau^{\prime}
$$

It is straightforward to show that we obtain Eq. (2.11) by simply applying Hamilton's equations. In this case, the canonical momentum $p$ is

$$
p=\frac{d \tau}{d t}=\frac{\Delta \omega}{\omega_{0}}=-\eta \epsilon
$$

So what is $V(\tau, t)$ that appears in Eqs. (2.11) and (2.12)? Neglecting synchrotron radiation, $V(r, t)$ is given by

$$
V(\tau, t)=V_{\mathrm{rf}}(\tau)+V_{W}(\tau, t)
$$

where $V_{\mathrm{r}}(\tau)$ is the integrated voltage gain for one turn due to the external RF. That is

$$
V_{\mathrm{rf}}(\tau)=\hat{V} \sin \omega_{\mathrm{rf}}\left(\tau-\tau_{0}\right)
$$

Here $\omega_{A}$ is the RF frequency and $\tau_{0}$ is the stable phase angle. In further equations we will set $\tau_{0}=0$. On the other hand, $V_{W}(\tau, t)$ is the wake potential. That is, it is the integrated voltage gain of a particle at $\tau$ and at time $t$ due to the fields induced by the beam in the surroundings (e.g., bellows, RF cavities, etc.) in one turn.

If we neglect $V_{W}(\tau, t)$, and examine the motion for small $\tau$, we find

$$
\frac{d^{2} \tau}{d t^{2}}+\frac{\eta e \hat{V} \omega_{\mathrm{rf}}}{E_{0} T_{0}} \tau=0
$$

In this case the motion in the longitudinal direction is simple harmonic with a frequency given by

$$
\omega_{s}^{2}=\frac{\eta e \hat{V} \omega_{\mathrm{r}}}{E_{0} T_{0}}
$$

If we look at the Hamiltonian for this motion, we simply expand and keep up to quadratic terms (dropping any constant terms) to find

$$
H=\frac{p^{2}}{2}+\frac{\omega_{y}^{2} \tau^{2}}{2}
$$

The Hamiltonian in Eq. (2.18) is a constant of the motion since it has no explicit time dependence. Now that we have found a constant of the motion for externally applied RF, it is useful to see how this is modified by the wake potential $V_{W}(\tau)$. 
Consider the case when the distribution of particles is stationary (not oscillating in time). In this case, the wake voltage is only a function of $r$ and not a function of $t$. From Eq. (2.12) we can write the Hamiltonian as

$$
H=\frac{p^{2}}{2}+\frac{\omega_{s}^{2} \tau^{2}}{2}+\frac{\eta e}{E_{0} T_{0}} \int^{\tau} V_{W}\left(\tau^{\prime}\right) d \tau^{\prime}
$$

which is again a constant of the motion. We see that the wake potential has modified the effective potential well in which the beam particles oscillate. Before discussing the effect of this potential well distortion, it is useful to discuss the wake potential.

\subsection{The Collective Force}

\subsubsection{The Longitudinal Wake Potential}

We are faced with a dilemma here. We need to know the bunch distribution in order to calculate the field that it induces in the environment. But in fact, those fields change the bunch distribution. The solution to this problem in the time domain is to calculate a Green function or wake potential and in the frequency domain to calculate an impedance function. Since the time domain and the frequency domain are useful for different applications, we must discuss both. Let us consider two particles traversing a structure at $v=c$ (see Fig. 2). The first particle of charge $Q$ induces a longitudinal electric field $E_{z}(z, t)$ along its path. The second particle behind a distance $\tau$ and also traveling at speed $c$ sees the field $E_{z}\left(z, \frac{z}{c}-\tau\right)$. Given this electric field, the wake potential per unit charge felt by the trailing particle is defined to be

$$
W_{z}(\tau)=\frac{1}{Q} \int E_{z}\left(z, \frac{z}{c}-\tau\right) d z
$$

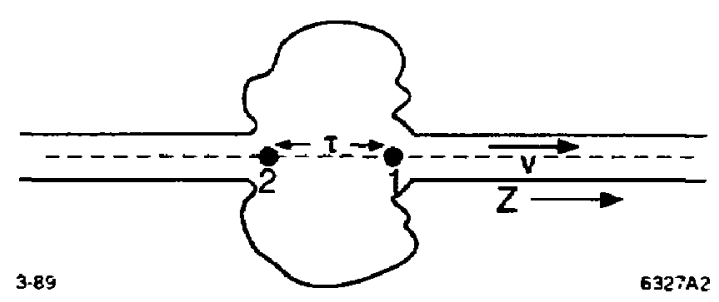

Fig. 2. Two partıcles passing through some structure on axis.

Note that the integral must extend wherever the field is non-zero. To add up the effects of a bunch of particles we simply use linear superposition. Let us consider a current distribution $I(r)$ 
defined so that

$$
\int I d \tau=e N=\text { Total Charge } .
$$

In this case, the voltage induced by the current distribution $I(\tau)$ is given by

$$
V_{W}(\tau)=\int_{-\infty}^{\infty} W\left(\tau-\tau^{\prime}\right) I\left(\tau^{\prime}\right) d \tau^{\prime}=\int_{\infty}^{-\infty} I\left(\tau-\tau^{\prime}\right) W\left(\tau^{\prime}\right) d \tau^{\prime}
$$

It is useful to note some properties of the wake function of $W(\tau)$. Since the particles are travelling at a velocity equal to $c$, then by causality the wake function $\tau$ must vanish ahead of the bunch, that is

$$
W(\tau)=0, \quad \tau>0
$$

There is one caution here. In the definition of the wake function, we have held the bunch rigid as it passes through the structure. In a linac this is strictly true for $v=c$. However, in a storage ring this is only true for a short structure. It is not true for long distances since $\boldsymbol{\eta} \neq 0$ and thus particles within the distribution can oscillate longitudinally. If, however, the acceleration voltage is sufficiently weak and the synchrotron frequency is sufficiently small, then it is possible to use this integrated wakefield to approximate the actual wakefield that the bunch would have as it travels through the structure.

\subsubsection{The Impedance}

Impedance is usually defined as the Fourier transform of the wake potential. That is

$$
Z(\omega)=\int_{-\infty}^{\infty} W(\tau) e^{+i \omega \tau} d \tau
$$

which means that the wake function can be expressed in terms of the impedance as the inverse Fourier transform

$$
W(\tau)=\frac{1}{2 \pi} \int_{-\infty}^{\infty} Z(\omega) e^{-i \omega \tau} d \omega
$$

The units of wake potential given here are volt/coulomb while the units of impedance are ohms. It is useful using the impedance to write the wake function due to a distribution of particles. To lo this, let us define the Fourier transform of the wake voltage $V_{W}(\tau)$ and the current distribution 
$I(\tau)$. These are given by

$$
\begin{aligned}
& V_{W}(\tau)=\frac{1}{2 \pi} \int_{-\infty}^{\infty} \tilde{V}(\omega) e^{-i \omega \tau} d \omega, \\
& I(\tau)=\frac{1}{2 \pi} \int_{-\infty}^{\infty} \tilde{I}(\omega) e^{-i \omega r \tau} d \omega .
\end{aligned}
$$

Thus, the full wake voltage is simply

$$
V_{W}(r)=\frac{1}{2 \pi} \int_{-\infty}^{\infty} d \omega Z(\omega) \tilde{I}(\omega) e^{-i \omega r}
$$

or, in other words, we have

$$
\tilde{V}_{W}(\omega)=\tilde{I}(\omega) Z(\omega)
$$

Thus, a convolution integral in the time domain has turned into a simple product in the frequency domain.

Now that we have expressed and defined the wake potential in terms of properties of the current distribution, it is time to return to study potential well distartion.

\subsection{Potential Well Distortion}

In Section 2.1, we found that the constant of the motion depended on the wakefield. Recall that

$$
\begin{aligned}
H & =\frac{p^{2}}{2}+\frac{\omega_{l}^{2} \tau^{2}}{2}+\frac{\eta \epsilon}{E_{0} T_{0}} \int^{\tau} V_{W}(\tau) d \tau^{\prime} \\
& =\frac{p^{2}}{2}+\frac{\omega_{s}^{2} \tau^{2}}{2}+\frac{\eta \varepsilon}{E_{0} T_{0}} \int_{-\infty}^{\infty} S\left(\tau-\tau^{\prime}\right) I\left(\tau^{\prime}\right) d \tau^{\prime}
\end{aligned}
$$

where

$$
S(\tau)=\int^{\tau} W\left(\tau^{\prime}\right) d \tau^{\prime}
$$

Since this Hamiltonian is not explicitly a function of the time, it it a constant of the motion. Now we must find out how this constant of the motion is related to the distribution of particles. To do this, consider a distribution $\psi(\tau, p, t)$, that is, a distribution which is a function of the coordinate, the canonical momenta and possibly the time $t$. Due to Liouville's theorem, the total 
time deriative of $\psi$ with respect to $t$ vanihes. That is

$$
\frac{d \psi}{d t}=0
$$

Expanding using the convective derivative we find

$$
\frac{\partial \psi}{\partial t}+\dot{r} \frac{\partial \psi}{\partial r}+\dot{p} \frac{\partial \psi}{\partial p}=0
$$

Eq. (2.32), known as the Vlasov equation, is the equation of motion for the smooth distribution in phase space given the equations of motion which determine $\dot{\tau}$ and $\dot{p}$ in terms of $\tau$ and $p$. The requirement for this to be a stationary distribution is simply that

$$
\frac{\partial \psi}{\partial t}=0
$$

When we express $\dot{r}$ and $\dot{p}$ in terms of the Hamiltonian we find

$$
\frac{\partial H}{\partial p} \frac{\partial \psi}{\partial \tau}-\frac{\partial H}{\partial \tau} \frac{\partial \psi}{\partial p}=0
$$

We would like to find the solution to this equation which is only a function of $\tau$ and $p$ and not an explicit function of the time $t$. As a guess, let us consider a function $\psi$ which is a function only of the Hamiltonian. That is, it is a function only of the combination of $r$ and $p$ which occurs in the Hamiltonian. We know, in fact, that this combination of $\tau$ and $p$ is an invariant. Using the chain rule, it is straightforward to show that in this case $\mathrm{Eq} .(2,34)$ is satisfied. However, we are far from finished. We have to close the system. First we select a normalization condition for $\psi\{r, p)$

$$
\iint \psi[H(\tau, p)] d \tau d p=1
$$

Then the longitudinal current distribution is given by

$$
I(\tau)=e N \int \psi[H(\tau, p)] d p
$$

Now if we substitute the expression for the Hamiltonian into the representation of the current in Eq. (2.36) we obtain

$$
I(\tau)=e N \int d p \psi\left[\frac{p^{2}}{2}+\frac{\omega_{s}^{2} r^{2}}{2}+\frac{\eta e}{E_{j} T_{0}} \int_{-\infty}^{\infty} S\left(\tau-\tau^{\prime}\right) I\left(\tau^{\prime}\right) d \tau^{\prime}\right]
$$

Even though we've specified that the distribution function is a function only of the invariant, we are ret done uritil we also satisfy the consistency condition in Eq. (2.37). That is the hard part. 
For an electron beam which is subject to radiation damping and quantum excitation, the bunch reaches an equillibrium distribution which is an exponential in the Hamiltonian. Thus, this yields a Gaussian distribution in p. This distribution is given by

$$
\psi=\frac{K}{\sqrt{2 \sigma} \sigma_{p}} \exp \left[-\frac{p^{2}}{2 \sigma_{p}^{2}}-\frac{\tau^{2}}{2 \sigma_{0}^{2}}-\frac{\eta \epsilon}{\omega_{,}^{2} E_{0} T_{0} \sigma_{0}^{2}} \int_{-\infty}^{\infty} S\left(\tau-\tau^{\prime}\right) I\left(\tau^{\prime}\right) d \tau^{\prime}\right]
$$

where

$$
\begin{aligned}
& \sigma_{p}=p_{\mathrm{rms}}=\eta \epsilon_{\mathrm{rms}}=\eta \frac{\left(E-E_{0}\right)_{\mathrm{rms}}}{E_{0}}, \\
& \sigma_{0}=\frac{\sigma_{p}}{\omega_{s}}=t_{\mathrm{rms}} \text { as } N \rightarrow 0,
\end{aligned}
$$

and $K$ is the normalizaticn factor. If we perform the integral in Eq. (2.37) and secall the definition of $w_{s}$, then we find the consistency condition for a Gaussian beam

$$
I(\tau)=e N K \exp \left[-\frac{\tau^{2}}{2 \sigma_{0}^{2}}-\frac{1}{\dot{V} \sigma_{0}^{2}} \int_{-\infty}^{\infty} S\left(\tau-\tau^{\prime}\right) J\left(\tau^{\prime}\right) d \tau^{\prime}\right]
$$

where $\dot{V}$ is $\omega_{\pi r} \hat{V} \cos \phi_{0}$.

To continue in this section it is useful to consider some examples of potential well distortion. ${ }^{5}$ Let's look at four examples; an inductive wake, a resistive wake, a capacitive wake, and a real wakefield from the SLC damping ring. That is, let's cunsider the wake voltage given by

$$
\begin{aligned}
& V_{W}=L \frac{d J}{d r} \\
& V_{W}=R I \\
& V_{W}=\frac{1}{C} \int^{r} I d r^{\prime} .
\end{aligned}
$$

The examples for each of the wakefields shown are given in Figs. 3, 4, 5, and 6. In Figure 3, you see the effect of an inductive wakefield at several values of intensity. For the lowest intensities the distribution is Gaussian and at the higher intensities it is parabolic for $y>>1$ and Gaussian at the tail. You see in Figure 3 that the bunch lengthens and becomes non-Gaussian because its full-width-at-half-maximum differs markedly from that for a Gaussian. In Figure 4, you see the effect of a resistive wake. Unlike the inductive wake, with the resistive wake the bunch centroid shifts back on the RF. This is due to the loss of energy with a resistive wake. The bunch must move on the RF so that it's supplied energy by the RF to make up this loss. Notice that the bunch length changes only rather slowly. 

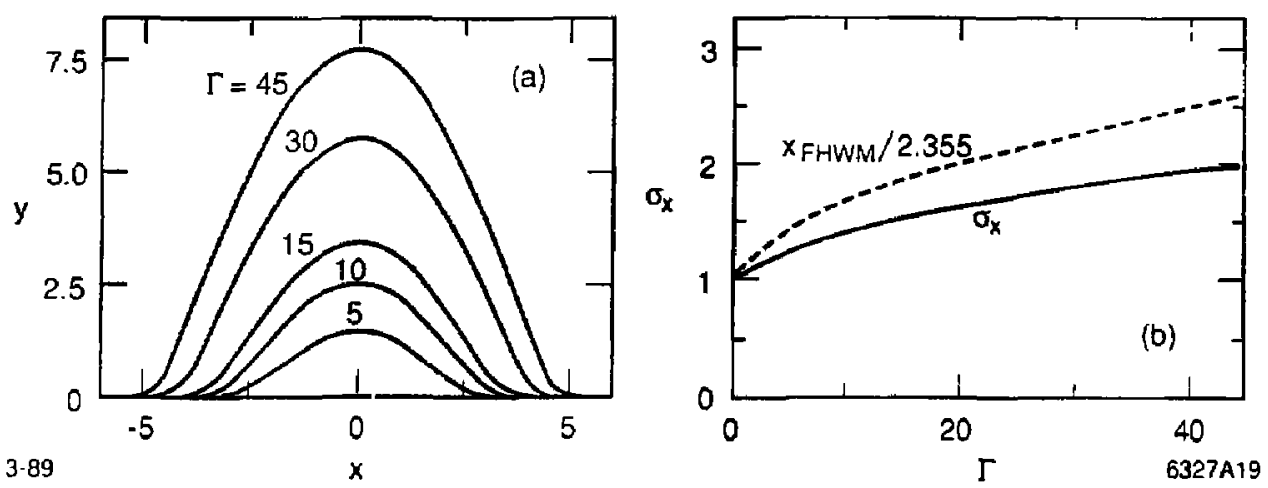

Fig. 3. Bunch shape changes for a perfect inductor, $y=L I /\left(\dot{V}_{\mathrm{r} f} \sigma_{0}^{3}\right), \Gamma=L e N /\left(\dot{V}_{\mathrm{rf}} \sigma_{0}^{3}\right), x=\tau / \sigma_{0}$.
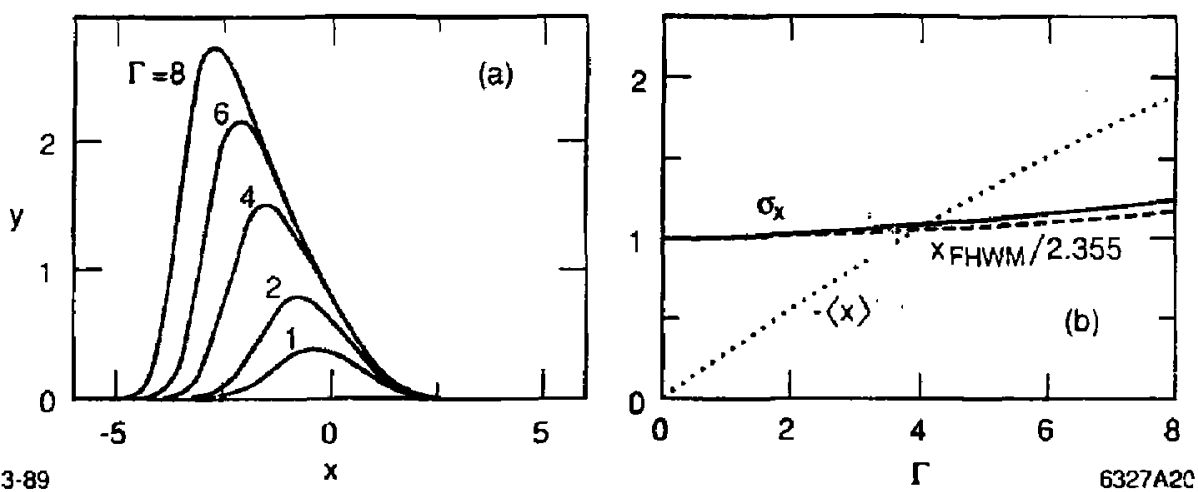

Fig. 4. Bunch shape change and centroid shifts for a perfect resistor, $y=R I /\left(\dot{V}_{\mathrm{r}} \sigma_{0}\right), \Gamma=R e N /\left(\dot{V}_{\mathrm{r}} \sigma_{0}^{2}\right), x=\tau / \sigma_{0}$.

For the capacitive wake in Fig. 5, we see a different situation. Since the capacitive wake is also lossy due to the charging up of the capacitor as the bunch goes by, the bunch centroid once again shifts back on the RF. However, the bunch length, in this case, actually shortens rather than lengthens. This is easy to understand due to the slope of the RF. For an inductive wake, the slope due to the wakefield is actually the opposite sign as that due to the external RF. This is because the example chosen is above transition energy. For capacitive wake the opposite is true. The slope due to capacitive wake function is actually the same sign as that for the RF; this leads to additional focusing and a shortened bunch length.

In Figure 6, we finally see bunch lengthening for the damping ring of the SLC. ${ }^{6}$ The damping ring wakefield is dominated by inductive and resistive components. You see that the bunch widens as well as the centroid shifting. However, the centroid doesn't shift simply linearly, it rolls over. The reason for this is that longer bunches tend to lose less energy while shorter bunches tend 

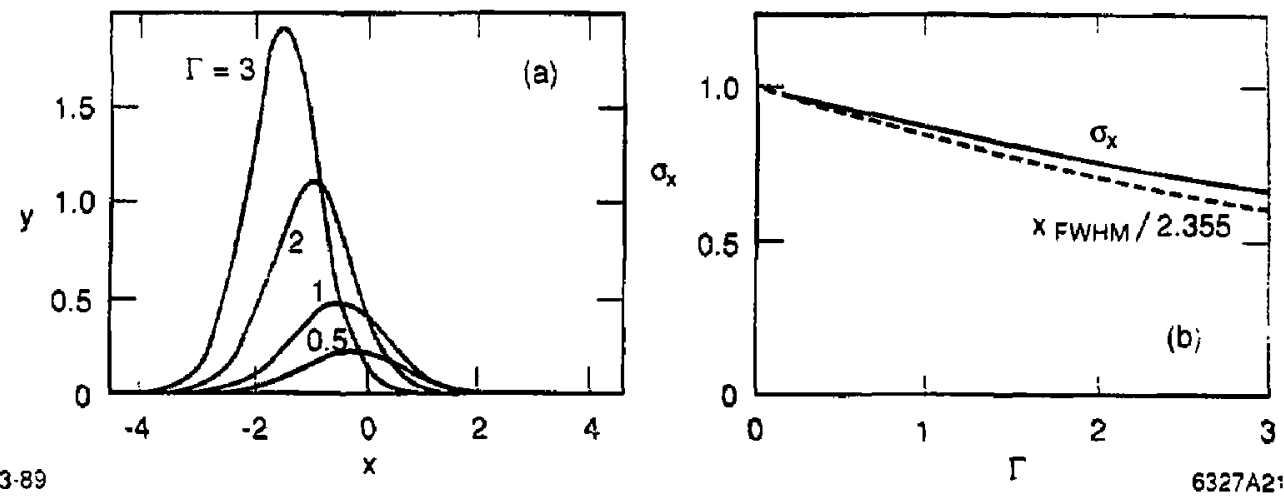

Fig. 5. Bunch shape change for a perfect capacitor, $y=I /\left(\dot{V}_{\mathrm{rf}} C\right), \Gamma=e N /\left(\dot{V}_{\mathrm{rf}} \sigma_{0} C\right), x=r / \sigma_{0}$.
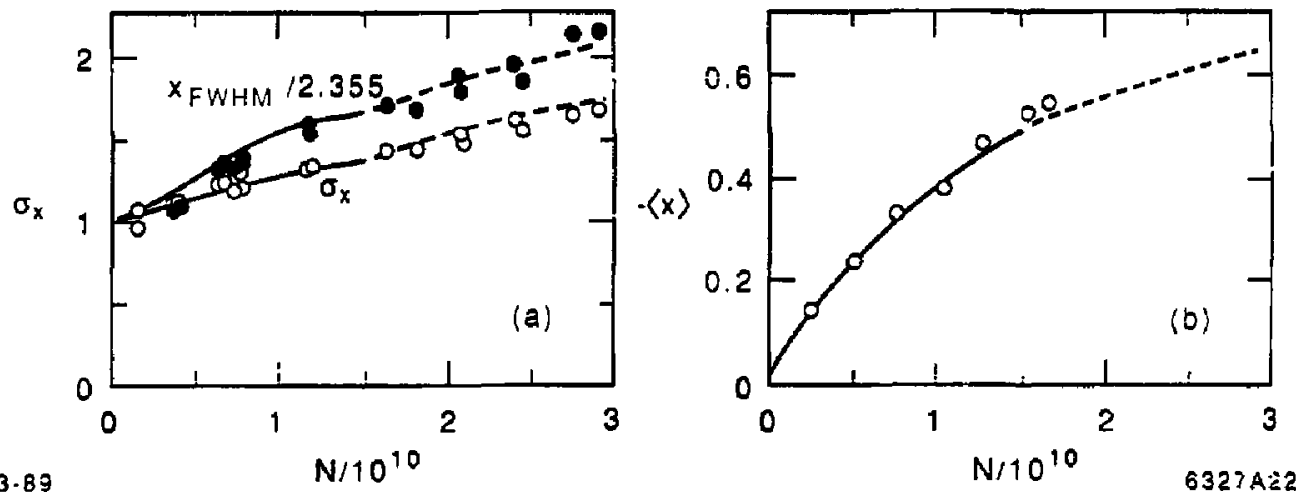

Fig. 6. Bunch lengthening and centroid shifts for the SLC damping rings.

Plotted points are data. Solid and dashed lines are calculations.

to lose more. Thus, the bunch lengthening which takes place due to the inductive part of the impedance actually decreases the amount of energy lost to the resistive part of the impedance.

The data in Fig. 6 are plotted as open or closed circles, and the lines correspond to a calculation of bunch lengthening using the calculated wakefield for the SLC ciamping ring. ${ }^{5,7}$ In Figure 7 you see a comparison of the distribution of particles obtained from measurements (open circles) to that obtained from the calculations using the damping ring wakefield. ${ }^{5,7}$ In both Figs. 6 and 7 , the agreement between the measurement and calculation is excellent.

In this section we have seen how the fields induced by a bunch of particles can affect the actual distribution of particles and how that distribution and field can be obtained in a self-consistent way. This leads to a change in the distribution function as the current is increased in a bunch of particles. We have not thus far discussed the question of whether this distribution is stable. Before contisuing to discuss the stability of the longitudinal distribution, we will turn now to the 


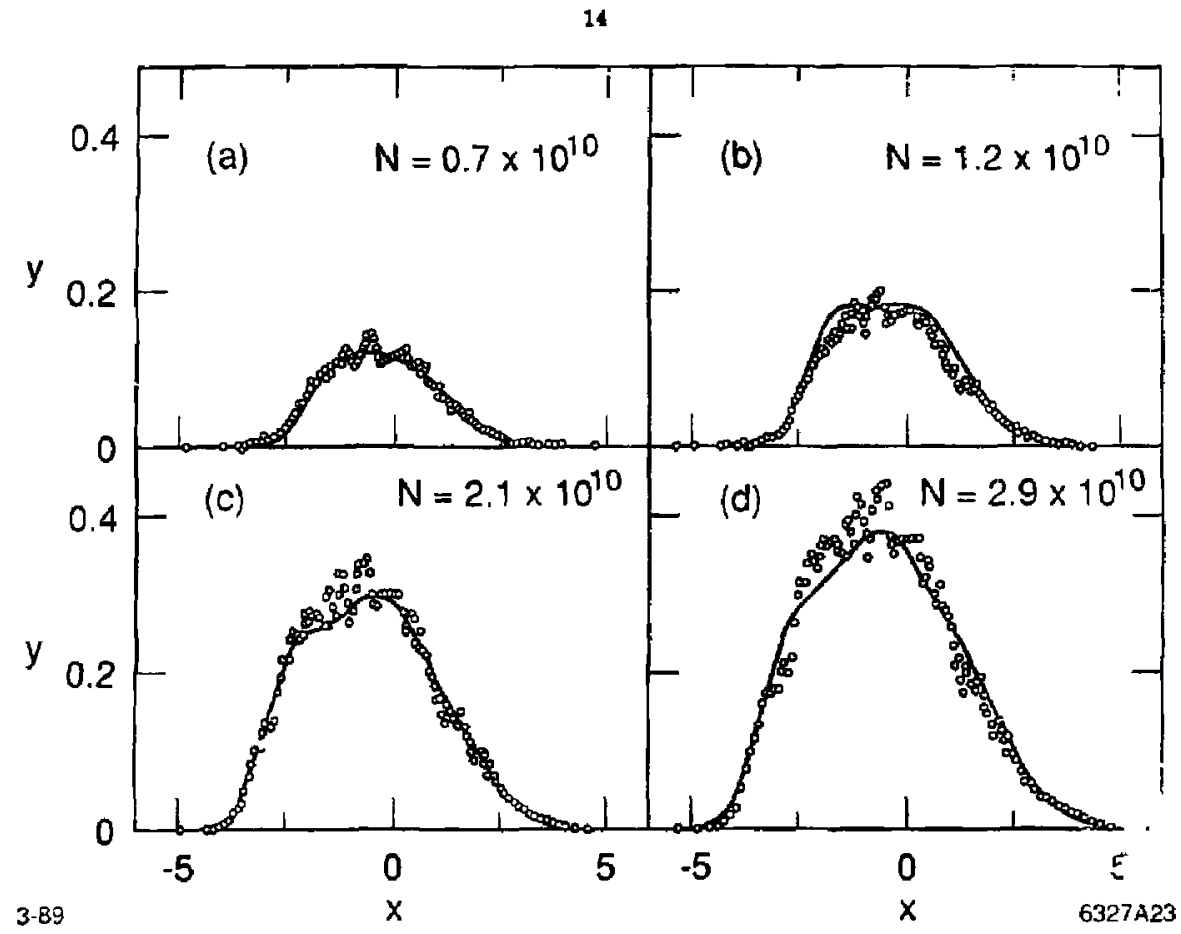

Fig. 7. Longitudinal bunch distribution for several values of intensity.

Plotted points are data; solid curves are calculations.

study of transverse motion to see the analogous effects in the transverse dimension.

\section{TRANSVERSE BASICS}

\subsection{EquaTIONS OF MOTION}

For a particle moving in a linear or circular accelerator focused by transverse magnetic fields, the equatios of motion for the transverse displacement is given by

$$
\frac{d^{2} y}{d s^{2}}+K(s) y=0
$$

where

$$
K(s)=\frac{e}{p c} \frac{\partial B_{z}}{\partial y}
$$

We include the effect of some coherent force $F_{c}$ on the right-hand side of the equation as follows:

$$
\frac{d^{2} y}{d s^{2}}+K(s) y=\frac{F_{c}}{\beta^{2} \gamma m c^{2}}
$$

In many cases it is useful to discuss the motion in terms of a smooth approximation. In this case, the focusing function $K(s)$ is replaced by a smooth focusing function $k^{2}$ which yields the average 
focusing effect of the lattice. In this case, the equation of motion becomes

$$
\frac{d^{2} y}{d s^{2}}+k^{2} y=\frac{F_{c}}{\beta^{2} \gamma m c^{2}}
$$

\subsection{The Collective Force}

The transverse wakefield for a structure is defined in a way analogous to that for the longitudinal wake. Consider two particles moving in a structure at $v=c$, but offset by an amount xo. (See Fig. 8.) The first particle of charge $Q$ induces a field $E_{\perp}(z, t)$ and $(\vec{v} \times \vec{B})_{\perp}(z, t)$. The second feels the transverse force which is evaluated at the time $t=z / c-\tau$. The wakefield is then defined as

$$
W_{\perp}(\tau)=\left.\frac{1}{L I_{0} Q} \int[\vec{E}+\vec{v} \times \vec{B}]_{\perp}\right|_{t=z / c-\tau} d z
$$

where $L$ is the length of integration. For small $L$ the transverse kick experienced by the trailing particle is

$$
x_{\text {before }}^{\prime}-x_{\text {after }}^{\prime}=\Delta x_{2}^{\prime}=\frac{e Q W_{\perp}(-r) x_{0} L}{E}
$$

Notice that the factor $L$ appears both in the definition in Eq. (3.5) and siso in Eq. (3.6). In some cases, this factor $L$ is included to obtain the wake force per unit length and in other cases it is excluded.

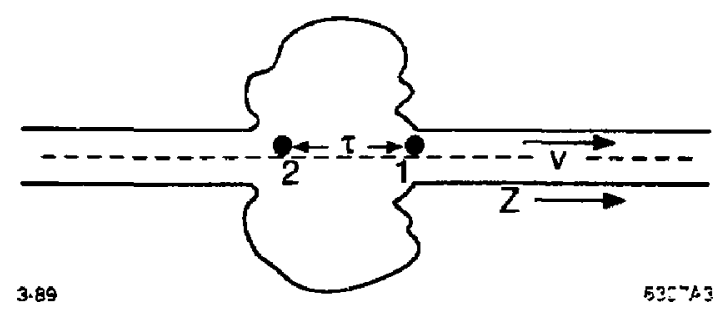

Fig. 8. Two particles offset from the center line by $x_{0}$ traversing a structure.

As in the longitudinal case, it is also useful to define a transverse impedance. In this case, we set the transverse impedance equal to the Fourier transform of the wakefield as follows

$$
Z_{\perp}(\omega)=-i \int_{-\infty}^{\infty} W_{\perp}(\tau) e^{+i \nu \tau} d \tau
$$

Notice that the factor of $-i$ has been included in the definition. This is conventional and is done so that it is the resistive part of the transverse impedance that causes instability. A transverse 
wakefield written in terms of the impedance is then given by

$$
W_{\perp}(\tau)=\frac{1}{2 \pi} \int_{-\infty}^{\infty} i Z_{\perp}(\omega) e^{-i \omega \tau} d \tau
$$

In this case, if we leave out the factor of $L$ in Eq. (3.5), the units for the transverse wake are volt/(coulomb $\mathrm{m}$ ) and the units for the transverse impedance are $\mathrm{ohm} / \mathrm{m}$. It is important and interesting to note that the transverse wakefield is rather different from the longitudinal. In Figs. 9 and 10 we see a comparison of the transverse wake and the longitudinal wake for the

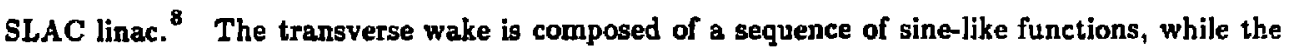
longitudinal wake is composed of a sequence of cosine-like functions.
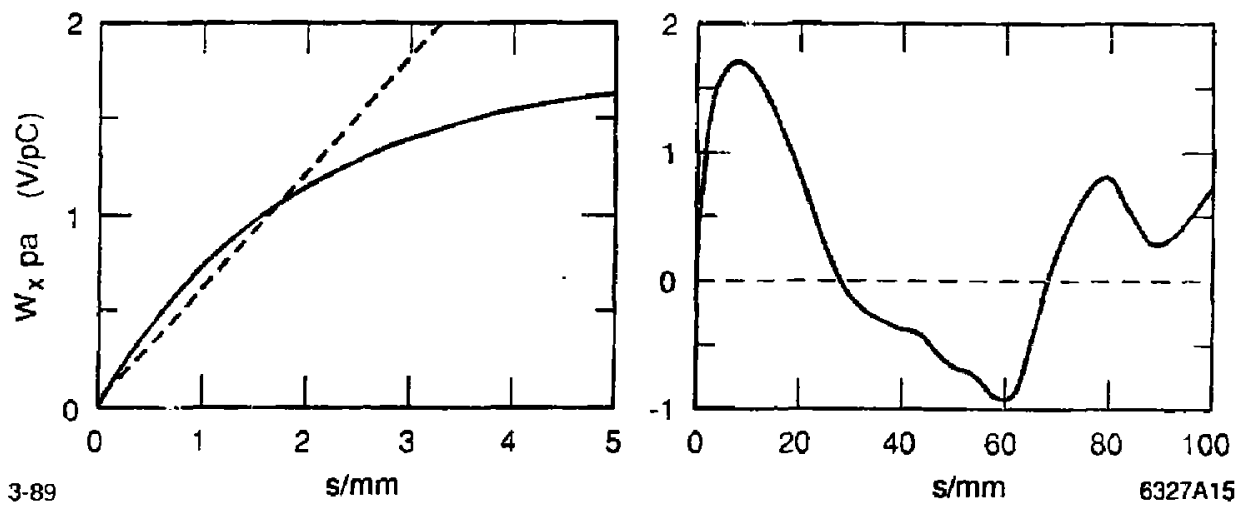

Fig. 9. Transverse wakefield per cell for the SLAC structure for a particle at the iris radius. The dashed line is a linear approximation to the wake seen by a $1 \mathrm{~mm}$ bunch.
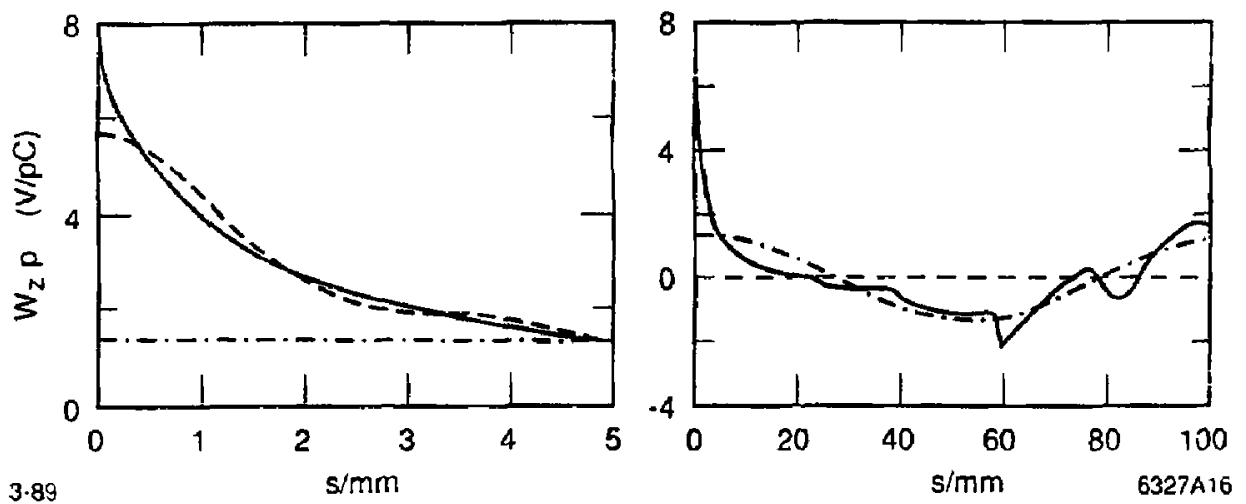

Fig. 10. Longitudinal wake per cell for an average SLAC cell. The solid curve includes an analytical extension for the high frequency behavior. 
Thus the longitudinal wake has a finite value as the separation between the test particle and the leading particle goes to zero, but the transverse wake goes to zero as the test particle approaches the leading particle. The effect of a transverse wake tends to decrease as the bunch length gets shorter, while the effect of the longitudinal wake tends to increase.

\subsection{Potential Well Distortion}

For a very high energy particle bunch, the transverse wake vanishes on axis. However, for low energy bunches there is a space-charge force due to the beam directly and also due to the image currents and charges in the wall. Let us consider a uniform distribution of charge transversely and longitudinally in the form of a cylinder and let us imagine that the walls of the vacuum environment are far away and let the cylinder have a radius a. Then the transverse force for a particle at transverse position $y$ is given by

$$
F_{y}=\frac{2 e^{2} \lambda y}{\gamma^{2} a^{2}}
$$

where $\lambda$ is the line density. This equation should be very familiar from calculations of static electric fields due to a cylindrical uniform line charge. The only difference is due to the factor of $\gamma^{2}$ in the denominator which arises from the cancellation of the electric and magnetic effects. Notice that in this case the field is purely linear within the charge distribution. Outside of the beam the field decays inversely with the distance away from the beam.

What is the effect of this space-charge force on the individual particles? In the previous sections, we discovered that we had to calculate a self-consistent distribution longitudinally. In this case, ideally, one must also find a self-consistent distribution. However, if the effect is small, that is if the space-charge forces are small compared to the externally applied field, then the distribution actually changes very little. However, in the transverse case in a circular accelerator, in spite of the fact that the transverse distribution changes little, the space-charge forces can have a profound effect on the single particle dynamics. If we recall the equation of motion, and substitute the space-charge force on the right-hand side then we find

$$
y^{\prime \prime}+K(s) y=\frac{F_{c}}{\beta^{2} \gamma m c^{2}}=\frac{2 r \lambda \lambda}{\beta^{2} \gamma^{3} a^{2}} y
$$

which for a longitudinally uniform beam becomes

$$
y^{\prime \prime}+K(s) y=\frac{r_{0} N}{\pi R \beta^{2} \gamma^{3} a^{2}} y
$$

where the line-charge density has been replaced by $\lambda=N /(2 \pi R)$, and $r_{0}$ is the classical radius of the particle considered. Since this is a linear term, it shifts the tune of the machine; that is, each particle in the beam will have the transverse oscillaticn frequency modified by the space charge 
effect of the whole beam. To calculate this effect, it is useful to recall the tune shift formula due to a small gradient perturbation in the circular accelerator. In this case, the tune shift is given by

$$
\Delta \nu \simeq \frac{1}{4 \pi} \int_{0}^{C} K(s) \beta(s) d s .
$$

Since the space-charge force is uniform around the ring, it is the average $\beta$ function which is the most relevant. If we let $\beta_{3 v e} \simeq R / \nu$, then the ture shift formula becomes

$$
\Delta \nu \simeq \frac{N R r_{0}}{2 \pi \nu} \frac{1}{\beta^{2} \gamma^{3}} \frac{1}{a^{2}}
$$

where $\nu$ is the unperturbed tune. Eq. (3.13) is called the Laslett tune shift. ${ }^{9}$ In a bunched beam, of course, we need to include an additional factor which takes into account that the loca! current is higher in a bunched beam than in a coasting beam. And in the actual case, we must also take into account the beam environment, that is, the actual vacuum chamber impedance. These modify the formula, but the basic scaling remains the same.

In addition, since most beams are not actually uniform, there is a tune spread rather than simply a tune shift. That is, the tune shift is actually a nonlinear function. In this case, particles in the tail will have very little tune shift while particles near the center of the beam would have a larger tune shift. Eq. (3.13) given above is then a very good estimate of the tune spread of the beam. If we have such a tune spread in a beam, then in order to avoid resonances, say integer and half integer resonances, it is necessary that $\Delta \nu \lessgtr \frac{1}{2}$. This is a limit on the accimulated current in proton accelerators. The typical solution to this problem is to inject at higher energy either with a higher energy proton linac or with a booster synchrotron. We see that the gain is quite rapid as the injection energy is increased because $\Delta \nu \propto \gamma^{-3}$.

Let us now summarize the results of the introductory sections on longitudinal and transverse dynamics of particles under the influence of their wakefields. In the longitudinal case, we found that the beam lengthens due to the influence of the wakefield. This is due to a change in the focusing forces experienced by the particles longitudinally and results in a redistribution of the charge. The analogous effect also happens transversely; however, the strengths of the finld which are tolerable transversely are small, because they lead to a tune spread in the beam whici :auses the beam to overlap resonances. The redistribution effects are important transversely in devices which have low energy electron beams, such as klystrons, or in transport systems for heavy ions.

Now that we have studied these incoherent effects on the stationary particle distribution, it is time to discuss the stability of these distributions to coherent perturbations. In the next sections, we begin the study of collective effects by starting with the longitudinal. 


\section{LONGITUDINAL EFFECTS}

\subsection{LiNEAR ACCELERATORS}

In this section, we consider the longitudinal effects due to the wakefield in a high energy electron or positron linac. In a high energy linac, the particles travel at $v=c$ and thus remain fixed longitudinally; therefore, there are no instabilities. However, the wakefield causes particles to lose more or less energy depending on their position in the bunch and depending on their position in a train of bunches. These effects can be compensated somewhat by using the slope of the external RF or the fill time of the accelerator structure. In the first section, we will consider the effects of the single bunch.

\subsubsection{Single Bunch}

For a single bunch in a linear accelerator, only the short wake is relevant. The wakefield causes particles to lose more or less energy depending on their position in the bunch. This effect can be compensated somewhat by using the slope of the external RF; however, this does yield some minimum energy spread for a given longitudinal charge distribution. To begin, we note that a particle within a bunch feels an acceleration due to the external field given by

$$
\frac{d E(\phi)}{d z}=e \hat{\mathcal{E}}_{z} \cos \left(\phi-\phi_{0}\right)
$$

where we have defined $\phi=0$ as the bunch center and $\phi=\phi_{0}$ as the peak of the RF voltage. Relating this to the variable $r$ defined earlier, we see that

$$
\phi=\omega_{\mathrm{rf}} \tau
$$

On the other hand, the decelerating feld due to the longitudinal wake is given by

$$
\begin{aligned}
\frac{d E}{d z} & =-\int_{0}^{-\infty} I\left(\tau-\tau^{\prime}\right) W\left(\tau^{\prime}\right) d \tau^{\prime} \\
\int_{-\infty}^{\infty} I(\tau) d \tau & =Q
\end{aligned}
$$

Since we know the wakefield and the density of particles at each point along the bunch, we can calculate the total energy loss or gain by the bunch. Using linear superposition, this is given by

$$
k_{\mathrm{tot}} Q^{2}=\int_{-\infty}^{\infty} I(\tau) \int_{0}^{-\infty} I\left(\tau-\tau^{\prime}\right) W\left(\tau^{\prime}\right) d \tau^{\prime}
$$

In many cases, the longitudinal wakefield is expressed as a sum of modes with loss factors. In 
this case, the longitudinal wake can be written

$$
\begin{array}{rlrl}
W_{\|} & =\sum_{n=0}^{\infty} 2 k_{n} \cos \left(\omega_{n} \tau\right), \tau<0 \\
& =0 & , \tau>0
\end{array} .
$$

Substituting using a Gaussian bunch together with Eqs. (4.4) and (4.5), we find that the total loss parameter is given by

$$
k_{\text {tot }}=\sum_{n=0}^{\infty} k_{n} e^{-\omega_{n}^{2} \sigma_{r}^{2}} .
$$

Thus the total loss parameter, $k_{\text {tot, }}$ is given by the sum of the loss parameters of all the modes weighted by the Fourier transform of the bunch distribution. In Figure 11, you see the total loss parameter us. bunch length for the SLAC linac. ${ }^{8}$ Notice that shorter bunches lose more energy due to the fact that they excite higher-order modes in the cavities and thus lose energy to those modes also. Another way of looking at this loss is in the time domain. Referring back to Figure 10 , we see that the longitudinal wakefield increases at shorter distances, and thus short bunches induce higher fields which cause more energy loss.

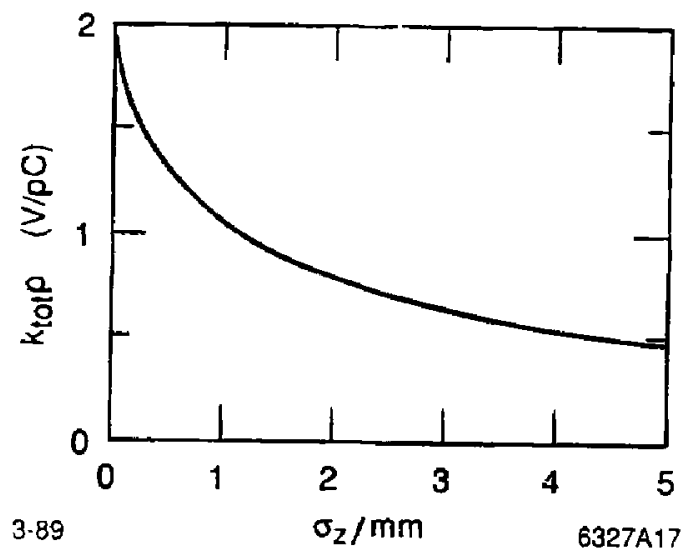

Fig. 11. The total loss parameter per cell vs. bunch length for the SLAC structure.

In addition to the energy loss, the wakefield causes an energy variation over the bunch. This can be approximately cancelled by variation of the phase on the RF by using the linear variation due to the RF to cancel approximately the variation due to the wakefield. However, this cancellation is not perfect. In Figure 12, you see calculations from Ref, 8 in which the RF phase has been optimized to yield the minimum energy spread. You see from Figure 12 that as a function of bunch length for the SLAC linac, the energy spread is minimum in the neighborhood 
of 1 to $1 \frac{1}{2} \mathrm{~mm}$. However, this depends on the amount of current in the bunch. In addition. the loss in acceleration gradient is also shown to be quite dramatic for shorter bunches due to the energy loss arguments discussed earlier. Therefore, for intense bunches, it is useful to have somewhat longer bunches so that the losses are less and the energy spreads are smaller.

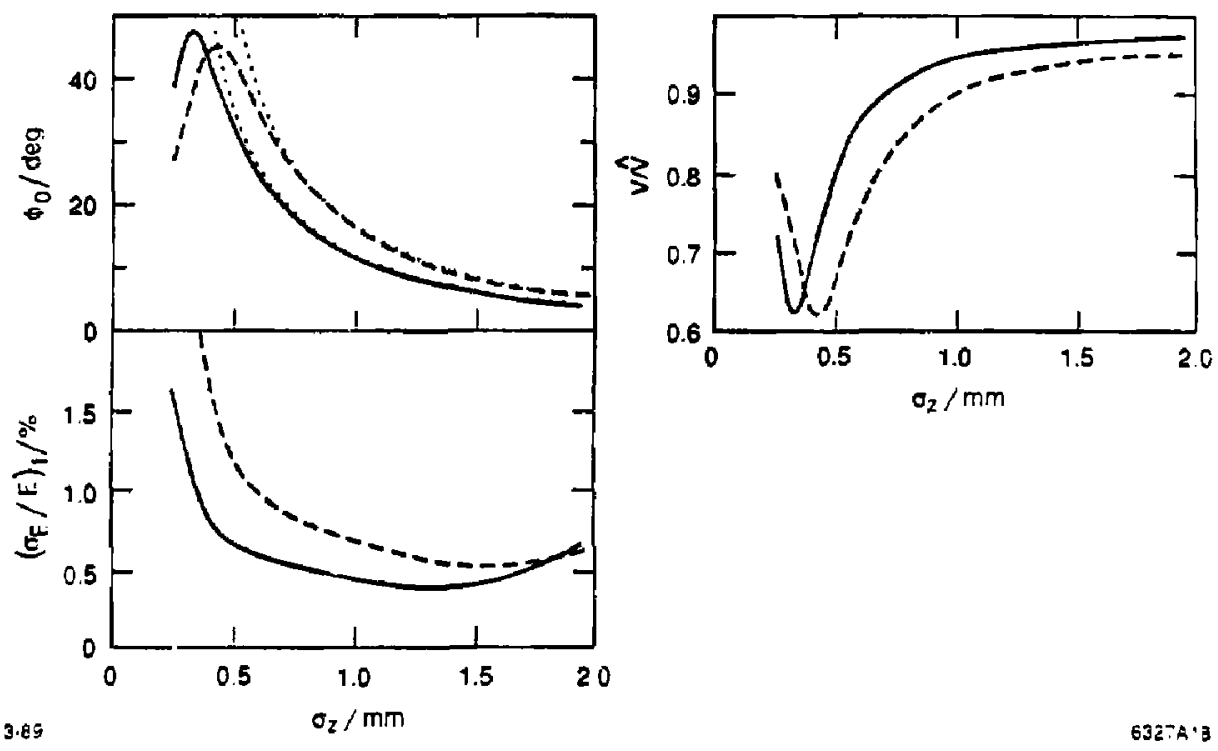

Fig. 12. SLC calculations: (a) Phase to minimize $\left(\sigma_{E} / E\right)$ final;

(b) the resulting final energy spread; and (c) relative loss of average acceleration gradient. $\hat{V}=17 \mathrm{MeV} / \mathrm{m}$ and $N=5 \times 10^{10}$ (solid) or $7 \times 10^{10}$ (dashes).

\subsubsection{Multi-bunch}

In a travelling wave linac, the first bunch through after filling sees the full accelerating field. Since it extracts energy, the second bunch sees less acceleration, and so forth. This can lead to a laige spread of energy from bunch to bunch unless measures are taken to prevent it. If the structure is partially filled, it is possible to match the rate of extraction with the rate of filling. To model this problem, let us consider the fundamental mode only. In this case, the wakefield is given by

$$
W_{i i}=2 k_{0} \cos \omega_{\pi} \tau
$$

Therefore, the field induced by a short bunch of charge $q$ is given by

$$
\frac{d E}{d z}=-2 k_{0} g \cos \omega_{\mathrm{rf}} \tau
$$




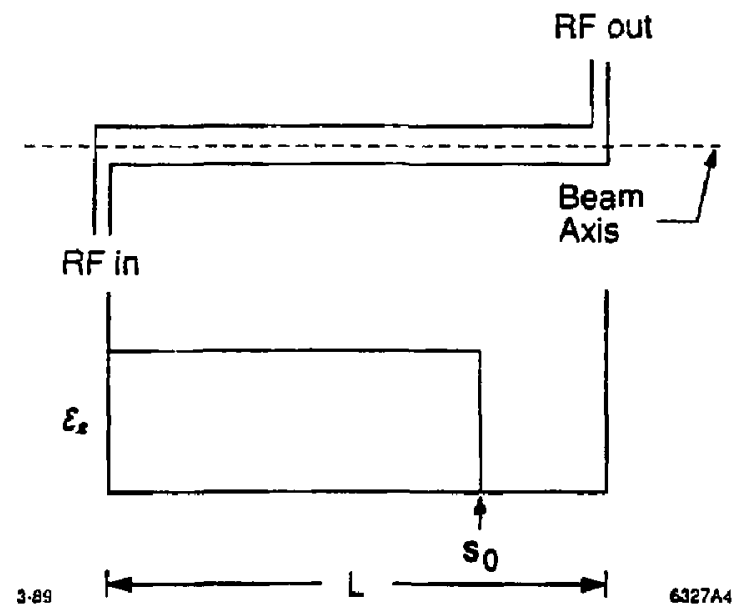

Fig. 13. Schematic of a partially full travelling wave RF structure.

To see the basic configuration, consider Figure 13 and a single bunch traversing an RF structure. Then the energy gain of a test charge at the front of the bunch is given by

$$
\Delta E=\int_{0}^{L} e \varepsilon_{z}(s) d s
$$

In Eq. (4.9), $\varepsilon_{2}(s)$ is the envelope of the RF as a function of distance along the structure. For the next calculations we assume the bunch to be at the crest of the cosine, and the cosine will be suppressed.

Now the problem is, after the first bunch has gone by, to keep the energy of all the bunches the same with some precision. To do this, let us consider the configuration in Figure 14. In this case, we have modelled the RF as a square wave entering a structure; the wakefield induced by the bunch as it travels through the structure is represented on the negative side of the axis to denote that it is decelerating. Just after the first bunch, the electric field has reached the point $s_{0}$ shown in Figure 14. The wakefield has filled the entire structure since the particle has traversed the entire structure. Just before the second bunch arrives, the wakefield has propagated out the end of the structure slightly, and the electric field has filled the structure somevhat more. To match the energy of the first and second bunch, one simply has to match the additional inflow of energy from the RF with the amount of wakefield induced by the first bunch. The situations just after the second bunch and just before the third bunch are also presented in Fig. 14. If we decide to match the energy of the first and second bunches then the energy of the third bunch will be off slightly. However, there is a possibility of approximately matching the energy of a long train of bunches by matching the first bunch to the tast bunch's energy gain. In order to do this, 
it is necessary to match the bunch spacing with the loading so that

$$
\frac{\Delta t}{T_{f}}=\frac{\eta_{0}}{2} \frac{1}{1+\frac{N}{4} \eta_{0}} .
$$

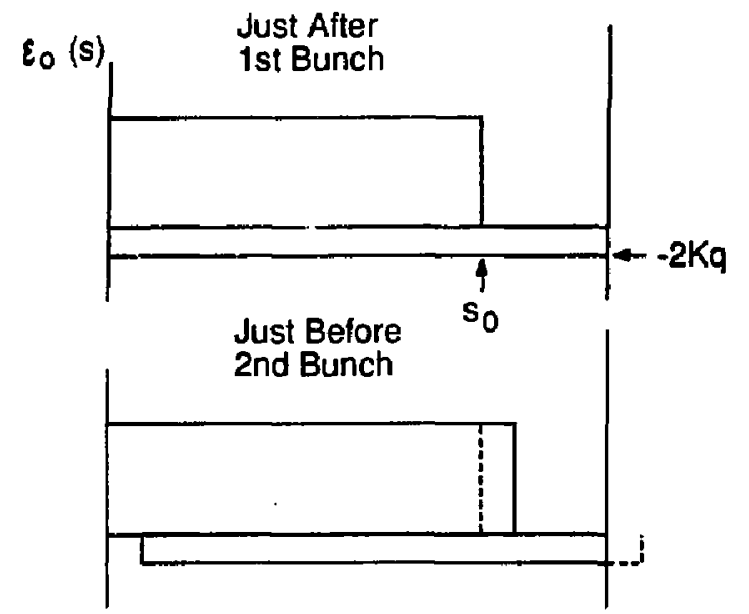

Just After

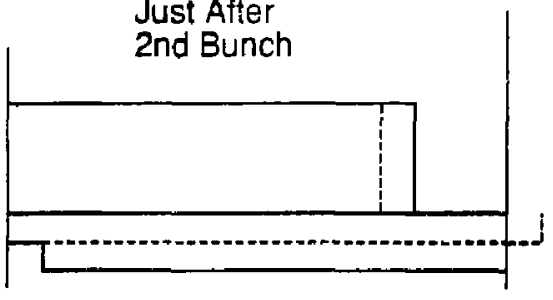

Just Before

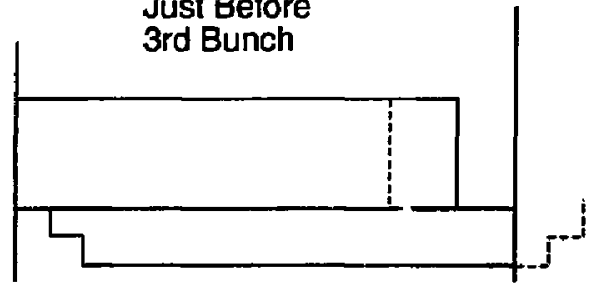

3-60

6327AS

Fig. 14. Schematic of the filling of the RF structure and the extraction of energy by two consecutive bunches.

Note that in Eq. (4.10) and all subsequent ones in this section, the losses in the traveling wave structure have been neglected. For the modifcations due to finite losses, see Ref. IU. In the middle of the bunch train there is an energy droop given by

$$
\frac{\delta E_{\text {पax }}}{E} \simeq \frac{N(N-2)}{32} \eta_{0}^{2}
$$

In addition, since the structure is only partially full, the average acceleration gradient has been 
reduced. This reduction is given by

$$
\frac{E_{\text {actual }}}{E_{\max }}=1-\frac{N \eta_{0}}{2},
$$

andi thus the overall efficiency is

$$
\eta_{N}=N \eta_{0}\left(1-\frac{N \eta_{0}}{2}\right)
$$

To illustrate this, let us consider the following example. If we set a tolerance on the maximum deviation and enerey from bunch to bunch to be

$$
\left(\frac{\Delta E}{E}\right)_{\operatorname{toax}} \simeq 10^{-3}
$$

and consider the number of bunches $N=10$ then this yields

$$
\eta_{0}=2 \%
$$

and the separation of the bunches in time is given by

$$
\frac{\Delta t}{T_{f}}=9.95 \times 10^{-3}
$$

Then if we consider an RF frequency of $17.1 \mathrm{GHz}$ and a fill time of $70 \mathrm{nsec}$ this yields a $21 \mathrm{~cm}$ : bunch spacing, which is 12 RF periods between bunches. In this case, the total efficiency of extraction is given by

$$
\eta N=18 \%
$$

Since the wakefields here are being balanced with the external RF, it is necessary that there be a tight tolerance on the number of particles. In particular, the total number of particles must have a variation less than about $1 \%$ in order to maintain the tolerance of $\Delta E / E$.

If the wakefield fundamental is cancelled as in the previous section to allow $\sim 20 \%$ beam loading, the short intense bunches will excite higher order modes up to $\omega \sim 1 / \sigma_{T}$. The cumlulative effect of this wakefield can lead to bunch-to-bunch energy variations; because of this, higher-order longitudinal modes may have to be damped.

From the previous section, the total loss parameter governs how much energy is radiated into a travelling wave structure,

$$
k_{\mathrm{tot}}=\sum_{n=0}^{\infty} k_{n} \mathrm{e}^{-\omega_{n}^{2} \sigma_{r}^{2}} .
$$

Since the bunches are so short, man? modes are excited and their relative importance is given by the $k_{n}$ 's. To illustrate the relative importance, the full wake calculated with modes up to $n=14$ 
for a disk-loaded structure at $11.4 \mathrm{GHz}$ is shown in Figure 15a. In Figure 15b, we have separated the fundamental mode from the higher modes to illustrate the relative importance. For this case, the energy variation due to higher modes ha been calculated by Palmer. "I For high loading the variation in beam energy was shown to be the order of $1 \%$ for about twenty bunches. However, it was found that if the longitudinal modes in the cavities could be damped to $Q$ 's of the order of 80 , the induced energy spread could be reduced by an order of magnitude to acceptable levels.
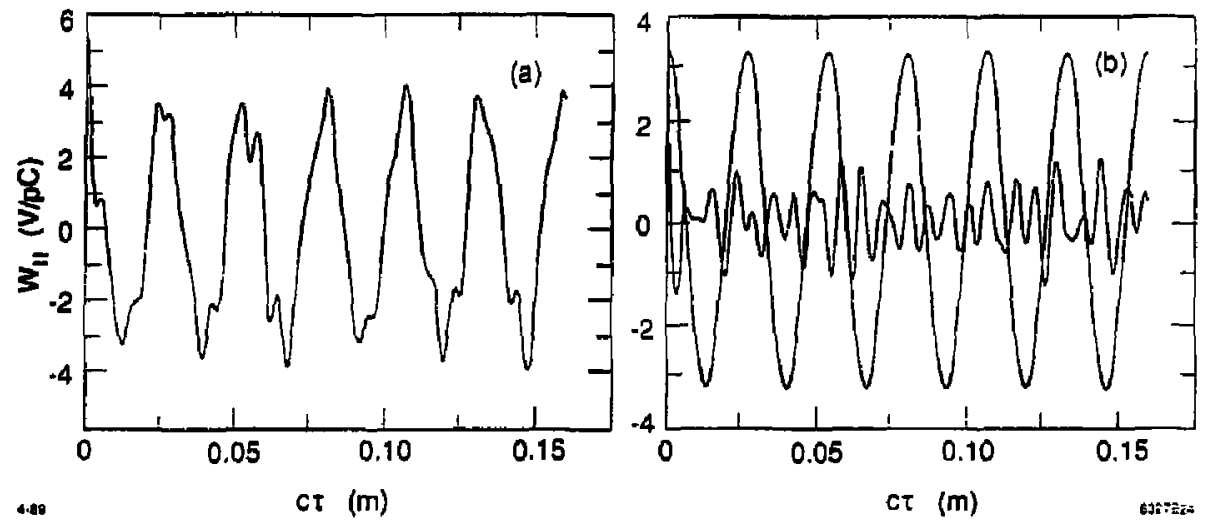

Fig. 15. Longitudinal wakefield for an $11.4 \mathrm{GHz}$ structure,

(a) The total wakefield; (b) the furdamental together with the higher modes.

\subsection{CirCular 4CCELERATORS}

\subsubsection{Single Bunch}

We have seen that potential well distortion produces changes in bunch length and shape. In an $e^{-}$ring above some threshold current, the energy spread also increases. This is due to a longitudinal instability. This instability has also been observed in proton rings, where it has been dubbed the microwave instability; however, in proton rings it is manifested somewhat differently due to the lack of radiation damping.

Due to the nature of this instability, it is useful to consider the physics of a coasting beam first. In this section, we will have a brief digtession on coasting beams.

\section{A. Coasting Beams}

Consider a coasting beam of current $I$ and energy $E$ circulating in a ring with revolution frequency $\omega_{0}$ and a frequency slip factor $\eta$. The coasting beam supports travelling wave modes 
given by

$$
I=I_{0}+I_{1} e^{i(n \theta-\omega t)}
$$

where $\theta$ is the azimuth, $n$ is the mode number (integer) and $\omega$ is the frequency in the lab. It is useful to go to beam coordinates; therefore, we let

$$
\phi=\theta-\omega_{0} t
$$

Rewriting Eq. (4.19) in terms of beam coordinates, we find

$$
I=I_{0}+I_{1} e^{i\left|n \phi-\left(\omega-n \omega_{\phi}\right) t\right|}
$$

thus, the frequency in a lab frame is given by

$$
\omega_{\text {lab }}=n \omega_{0}+\Omega_{\text {beam }}
$$

Now consider a longitudinal impedance $Z(\omega)$, and let the beam have a zero momentum spread. Then it is straightforward to show ${ }^{12}$ that

$$
\Omega= \pm\left[\frac{i \eta \omega_{0}^{2} e \operatorname{In} Z\left(n s_{0}\right)}{2 \pi \beta^{2} E}\right]^{\frac{1}{2}}
$$

There are several consequences of Eq. (4.23). Let's consider them on a case by case basis.

Case $1: \operatorname{Re}(Z) \neq 0$. In this case there are two modes, one which is damped and one which is anti-damped; thus, there is an instability.

Case 2: $\operatorname{Re}(Z)=0, \operatorname{Im}\left[Z\left(n \omega_{0}>0\right)\right]<0(Z$ inductive). In this case, if the frequency slip factor $\eta>0$ (above transition energy), we have stability since we have two modes each with a real frequency. On the other hand, if $\eta<0$ then we have an instability, one mode being stable and the other unstable.

Case 3: $\operatorname{Re}(Z)=0, \operatorname{Im}\left[Z\left(n \omega_{0}>0\right)\right]>0(Z$ capacitive $) . \quad$ In this case, if the frequency factor $\eta>0$, the beam is unstable with one mode unstable and other stable. On the othes hand, if $\boldsymbol{\eta}<0$, then the beam is stable, both modes having only a real frequency shift.

It is interesting to note that Case 3 with $\eta>0$ is the negative mass instability. Normally: a capacitive impedance, which is like space charge, is stabilizing. If a lump forms in a bunch, the particles in the front of the lump are repelled forward and those at the tail of the bunch are repelled backward, and thus the lump tends to smooth itself out. On the other hand, if the frequency slip factor $\boldsymbol{\eta}>\mathbf{0}$ (above transition energy), then the opposite happens. If a lump forms, particles at the head of the bunch are accelerated. However, since higher energy particles 
take longer to go around, they slip back in the bunch, and thus the lump tends to grow instead of io dissipate; this causes an instability. It is also interesting to note that the negative mass instability is the reason that Saturn has smooth rings in spite of the fact that gravitational force is an attractive force.

Returning now to coasting beams, fortunately, most beams do not have a zero moment um spread. An additional spread in momentum yields a spread in revolution frequency which causes Landau damping. ${ }^{13}$ The relevant spread in frequency is given by

$$
\Delta w_{\text {lab }}=n \Delta w_{0}=n \frac{\Delta \omega_{0}}{\omega_{0}} \omega_{0}=n w_{0}\left(n \frac{\Delta p}{p}\right)
$$

The relative size of the shift in frequency and the spread of frequency determines whether or not a beam is Landau damped. When the shift in frequency is less than the spread in frequencies there is stability; when the shift in frequencies is more than the spread there is instability. This implies a threshold condition given by

$$
|\Omega|<n w_{0} \eta \frac{\Delta p}{p}
$$

If we square the threshold condition, we find ${ }^{12}$

$$
\frac{e I\left|Z\left(n \omega_{0}\right) / n\right|}{2 \pi E \eta(\Delta p / p)^{2} \beta^{2}}<1
$$

For a Gaussian beam we let

$$
\frac{\Delta p}{p} \rightarrow\left(\frac{\Delta p}{p}\right)_{\operatorname{rms}}=\sigma_{e}
$$

and for a Lorentzian line shape we let

$$
\frac{\Delta p}{p} \rightarrow\left(\frac{\Delta p}{p}\right)_{\text {hwhm }}
$$

Thus we find that for currents up to some threshold value, the beam is stable due to Landau damping; but for those currents above the threshold value, the particles can indeed cooperate coherently and yield instability.

\section{B. Fast Blow-up of Long Bunches}

Now let us return to bunched beams to see if the coasting beam physics which was just discussed applies there. What are the differences between the bunched beam and the coasting beam? In the first place, particles circulate longitudinally with frequency $w_{0}$. In a bunch of particles, approximately one half of the particles have revolution frequency greater than the nominal revolution frequency; the other half have revolution frequency less than that. The particles circulate in longitudinal phase space causing the selative frequency difference to oscillate. Therefore, 
on the average the frequency spread is zero. In addition, the particle density is lumped in the bincfici bearn. Thus, the plane waves which were used in the analysis for coasting beams are not adequate. We need a wave packet to describe a lumped particle density distribution. In spite o: $\quad \therefore$ : $\because$ gyences, it is possible to have coasting-beam-like instability in a bunched beam under tre foicinisg conditions.

1. The growth rate must be fast compared to $w_{8}$. That is, the instability takes a snapshot of the bunch and the instantaneous relative momentum spread. Therefore, during the instability $\Delta \omega_{0} / \omega_{0} \neq 0$.

2. The impedance must be broad band relative to the bunch spectrum (Fourier transform of the line density). Actually, this is not an essential part of the restriction; but it makes the formula simple and analogous to those for a coasting beam.

3. The instability must occur at wavelengths short compared to a bunch length.

If all these are true, the middle of a bunch cannot tell that it is not part of a long coasting beam; thus, it is possible for a local instability to occur within the bunch, a coasting-beam-like instability. For the threshold condition for a bunched beam under the previous conditions, we simply replace the current in the coasting beam formula by the peak current in a bunched beam to obtain

$$
\frac{e I_{\text {pakk }}\left|Z\left(n \omega_{0}\right) / n\right|}{2 \pi E n \sigma_{e}^{2}} \leq 1
$$

This equation is the Boussard conjecture, ${ }^{14}$ which was proved by Wang and Pellegrini ${ }^{15}$ to apply if conditions 1-3 are satisfied. However, it has a different interpretation than the normal threshold condition. It is a sufficient condition for no fast-blow-up in the bunch. If we substitute the peak current for a Gaussian line density, then we have

$$
\frac{e^{2} N\left|Z\left(n \omega_{0}\right) / n\right|}{\sqrt{2 \pi} \sigma_{\tau} 2 \pi E \eta \sigma_{\epsilon}^{2}}=F
$$

In Eq. (4.30), we have substituted a form factor in order to make this into a necessary and sufficient condition for instability. Of course $F \geq 1$. For an $e^{ \pm}$ring, the competition between radiation damping plus instability yields an equilibrium at an increased energy spread such that the threshold condition is satisfied. Provided that $\sigma_{\tau} \propto \sigma_{\epsilon}$, then

$$
\left.\Rightarrow \frac{c}{\sigma_{1} \cdot(N)} \bar{N}_{\mathrm{th}}\right)=\left(\frac{N}{N_{\mathrm{th}}}\right)^{\frac{1}{3}} .
$$

To illustrate the utility of the previous theory, let's consider an experiment done at the SLAC damping ring ${ }^{6}$ with the results shown in Fig. 16. In Figure 16a, you see the energy spread 
cubed plotted us. the bunch intensity. Note that above the threshold for instability at a value of about $1.5 \times 10^{10}$ per bunch, the cube of the energy spread increases linearly with bunch intensity, thus confirming the $1 / 3$ power loss dependence in Eq. (4.31). In Fig. 16b, c, d, yo: see data for the SLAC damping ring for relative energy spread vs. bunch intensity, tunch ler n.: us. bunch intensity, and the phase shift $v$ s. bunch intensity. The curves plotted with the data are calculations based on the damping ring wakes and include potential well distortion and the instability threshold. The dashed lines refer to calculations above threshold. These lines are nut fits to data but were actual calculations based on a calculated impedance of $\mathrm{K}$. Bane., ${ }^{5,7} \mathrm{Tl}: t$ agreement both above and below threshold is excellent.
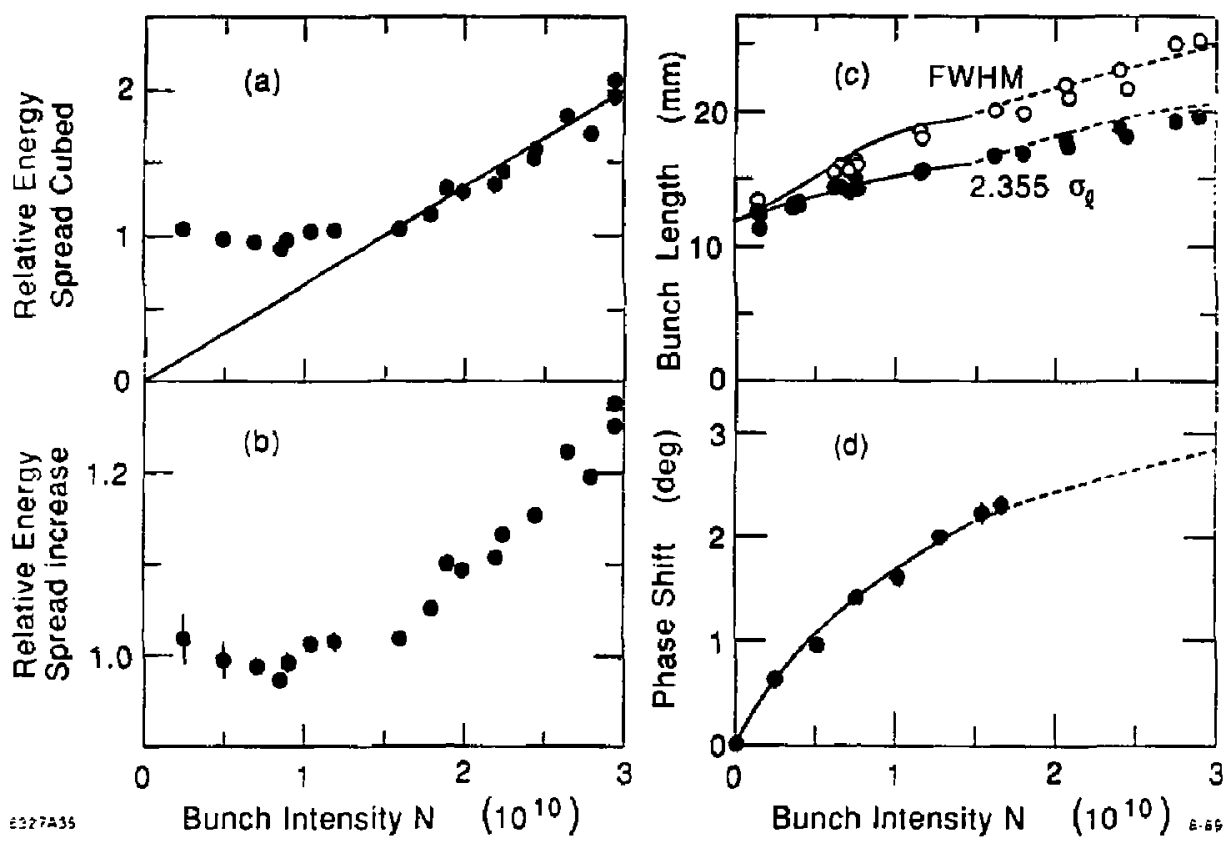

Fig. 16. Data and calculations from bunch lengthening experiments on the SLC damping rings. Plotted points are data, lines are calculations.

\section{Mode Coupling}

Below the threshold for fast blow-up of a bunched beam, the beam might be stable or have only' slow growth. For small enough current, the beam oscillates stably at frequencies $\boldsymbol{\omega}=n \omega_{0}+m \omega_{s}$. The circulation of the particles in longitudinal phase space keeps the beam stable. As you see from $E q$. (4.23), for a coasting beam there are stable and unstable modes depending upon the sign of $n$. For the bunched beam the coupling of these revolution modes results in stability. However, it is possible for an instability to occur if the frequencies of two synchrotron side bands become equal. (Note that the analysis of the coasting-beam-like instability for a bunched beam previously 
discussed includes modes of all $\mathrm{m}$.) Let's see what type of results we obtain if we include unly a few of the lowest-order modes of oscillation.

Let's consider including $m=1$ and $m=2$, dipole mode and quadrupole mode Thich ih. variation vs. current might look like Fig. $17 .^{16}$ Mode $m=2$ shifts down and couples $k m=1$. causing the instability shown. For a highly inductive impedance, we have

$$
\left|\frac{Z}{n}\right|=\omega_{0} L
$$

where $L$ is the inductance.
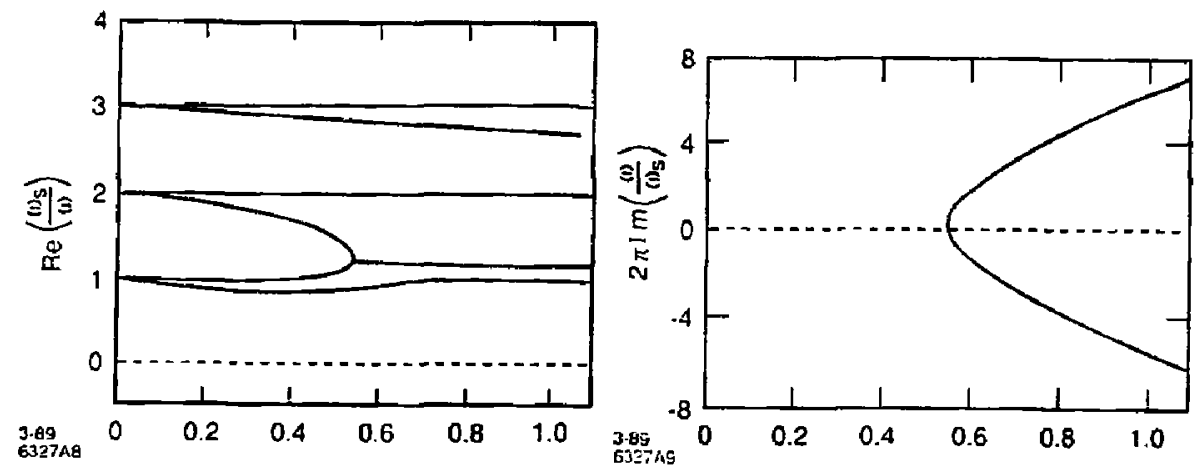

Fig. 17. Longitudinal mode coupling vs. current as calculated in Ref. 16.

If we estimate naively when the quadrupole mode and the dipole mode would collide for an inductive impedance, we obtain

$$
\frac{e^{2} N|Z / n|}{\sqrt{2 \pi} \sigma, 2 \pi E \alpha \sigma_{\epsilon^{2}}}>6 \sqrt{2} .
$$

The dipole mode does not shift, and the slope of the quadrupole mode is given by

$$
\frac{\Delta \omega_{s 2}}{\omega_{s}}=\frac{e^{2} N|Z / n|}{2 \pi E \alpha \sigma \epsilon^{2} \sigma_{\tau} 16 \sqrt{\pi}} .
$$

You see from Eq. (4.33) that for a highly inductive impedance, the two modes would nominally collide at a current much greater than the threshold for the microwave instability. Let us see how the initial slope compares with the data measured at the SLC damping ring. In this case, you see in Fig. 18 the data from Ref. 6 . Both the dipole mode and the quadrupole mode divided by 
2 are plotted on the same graph. The measured initial slope is given by

$$
\frac{d f_{\Delta 2}}{d N}=\frac{14 \mathrm{KHz}}{\left(10^{10}\right)}
$$

while that calculated with Eq. (4.34) is given by

$$
\frac{d f_{s 2}}{d N}=\frac{16 \mathrm{KHz}}{\left(10^{10}\right)}
$$

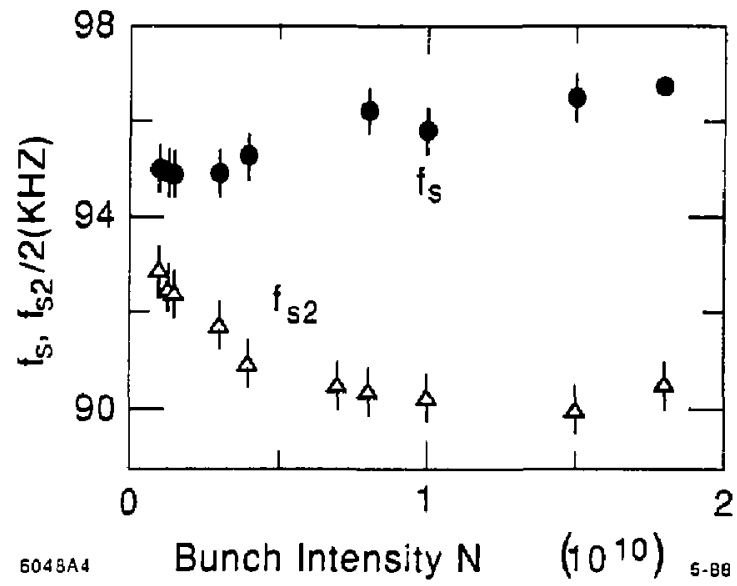

Fig. 18. Measurement of the shift of $m=1\left(f_{s}\right)$ and $m=2\left(f_{s 2} / 2\right)$ for the SLC damping ring.

Thus there is excellent agreement between the calculation and the data. As you see at an intensity of around $5 \times 10^{9}$, the quadrupole mode frequency stops its linear variation and begins to saturate. This is due to potential well bunch lengthening. As we see in Eq. (4.34), the slope of the quadrupole mode is sensitive to the value of the bunch length, and thus it begins to deviate from its approach to the dipole mode. It should be obvious from these results that the instability viewed in the SLC damping ring which begins at $1.5 \times 10^{10}$ cannot possibly be due to lower-order modes colliding because they simply have not moved enough to interact.

\subsubsection{Multi-bunch}

\section{A. Symmetric Coupled Bunch Instabilities}

In this section, we move to the study of the instabilities that are caused by the coupling between bunches. Consider a train of bunches equally spaced in a circular accelerator or storage ring. The coupling of one bunch to the next and from turn to turn via the long-range wakefield 
causes modes of oscillation that were discussed earlier ( $m=1,2$, etc.) to depend upon the other bunches. This coupling of modes can lead to instabilities in the train of bunches. To illustrate this, we will consider only dipole oscillations of each individual bunch coupled together. Consider, for example, two bunches located on opposite sides of a storage ring. In this case, there are two modes of oscillation. In the 0 mode bunches oscillate rigidly in a dipole fashion in phase with each other; in the $\pi$ mode ascillation, the bunches oscillate out of phase with each other. In this case, the coherent frequency shift is given by

$$
\frac{\Delta \Omega_{ \pm}}{\omega_{s}}=\frac{i I_{B}}{2 h \hat{V}_{d g} \cos \phi_{0}} \sum_{n=1}^{\infty}\left(1 \pm(-1)^{n}\right) n\left[Z\left(n \omega_{0}+\omega_{s}\right)-Z^{\prime}\left(n \omega_{0}-\omega_{s}\right)\right]
$$

where $I_{B}$ is equal to current/bunch and $h$ is the harmonic number. In Eq. $(4.37)+$ refers to the 0 mode and - refers to the $\pi$ mode. Note that the 0 mode couples only to even harmonics of the impedance, and the - mode couples only to odd harmonics.

Now let's consider an example of a particular impedance. Let the impedance be due only to the fundamental mode in the cavity of the RF system. And let us assume the harmonic number is even, that is, $\omega_{\text {cav }} \simeq h \omega_{0}$ where $h$ is even. In this case, for the $\pi$ mode, $\Delta \Omega=0$; the $\pi$ mode is stable. If the bunches start out in a mode oscillation, they oscillate continuously at the frequency $\omega_{s}$ out of phase with one another, but their amplitudes do not grow with time. For the 0 mode and this simple impedance, we find the frequency shift

$$
\frac{\Delta \Omega_{+}}{\omega_{s}}=\frac{i I_{B}}{\hat{V}_{\mathrm{ff}} \cos \phi_{0}}\left\{Z\left(h \omega_{0}+\omega_{s}\right)-Z^{*}\left(h \omega_{0}-\omega_{s}\right)\right] .
$$

In our case, instability corresponds to a positive imaginary part of $\Delta \Omega$. To examine the stability, we simply need to take the imaginary part of Eq. (4.38) to obtain

$$
\operatorname{Im} \frac{(\Delta \Omega)}{\omega_{s}}=\frac{I_{B}}{\hat{V}_{\mathrm{rf}} \cos \phi_{0}}\left\{\operatorname{Re}\left(Z\left(h \omega_{0}+\omega_{s}\right)-\operatorname{Re}\left[Z\left(h \omega_{0}-\omega_{s}\right)\right]\right\}\right.
$$

From Eq. (4.39), there are two distinct cases. First, consider the case for $\eta>0$ (above transition). In this case, $\cos \phi_{0}>0$. Then for $\omega_{\text {cav }}<h \omega_{0}$ the mode is damped as we can see from Fig. 19, since the imaginary part of $\Delta \Omega$ in this case is less than 0 . On the other hand, if $\omega_{c a v}>h \omega_{0}$ then we have instability since the imaginary part of $\Delta \Omega$ is greater than 0 . This is called the Robinson instability or in the case of damping is called Robinson damping. ${ }^{17}$ Physically, when the bunch is on the high energy side of its synchrotron oscillation, we would like it to lose a bit more energy. Since for $E>E_{0}, \omega<\omega_{0}$, we want more resistance just below $\omega_{0}$ for the bunch to lose more energy. Of course, the situation is simply reversed bclow transition.

What about a more complicated impedance? In this case, the $\pi$ mode can indeed be unstable; and if we measure the usstable motion and Fourier analyze jt, then we will see side bands around the odd harmonics in frequency space. That is, the formula for the instability tells us what we will measure. 


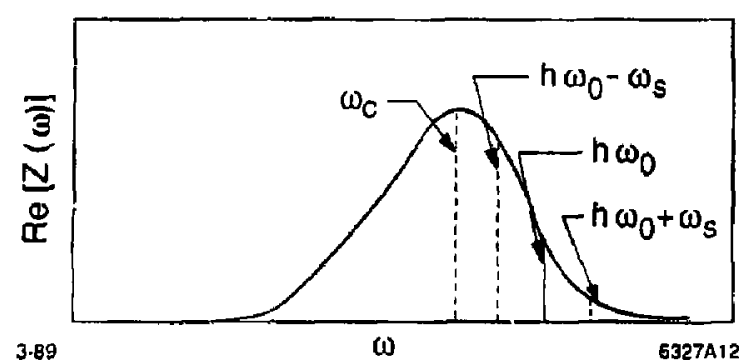

Fig. 19. The real part of the longitudinal impedance, illustrating the shift in frequency of the RF which yields Robinson damping.

In spite of the fact that Eq. (4.37) may preditt instability, it is possible that the beam be stable via Landau damping. Due to the nonlinear nature of the $\mathrm{RF}$, there is a spread in synchrotron frequency given by

$$
\frac{\Delta w_{f}}{\omega_{s}} \simeq \frac{1}{16} \omega_{\mathrm{rg}}^{2} \sigma_{f}^{2}
$$

If this spread is larger than the $\Delta \Omega$ shown in Eq. (4.37), then the beam may be Landau damped; and in this case, we have the typical threshold behavior of a Landau damped system.

\section{TRANSVERSE EFFECTS}

\subsection{SINGLE BUNCH}

\subsubsection{Linear Accelerators}

\section{A. Beam Break-up and BNS Damping}

In an $e^{ \pm}$linac, the transverse wake due to particles at the head of the bunch can cause the defiection of the tail. If the transverse betation oscillation frequency or wave number is the same for the head and tail, this can lead to resonant growth.

To model the problem, let us consider a two-particle model as shown in Fig. 7. We place one half of the charge in the bunch into each macro-particle and separate the particles by a distance $\ell$ which should be set to about $2 \sigma_{z}$ when compared to actual bunch distributions. The distance between the particles is fixed since they both travel at the speed of light; therefore, the wakefield at the trailing particle is fixed. The equations of motion for the two particles in the presence of the external focusing system are

$$
\begin{aligned}
x_{1}^{\prime \prime}+k^{2} x_{1} & =0 \\
x_{2}^{\prime \prime}+(k+\Delta k)^{2} x_{2} & =\frac{e^{2} N W(\ell) x_{1}}{2 E},
\end{aligned}
$$

where $N$ is the total number of particles in the two macro particles, and $W(\ell)$ is the wakefield at the second particle. 
Notice that the external focusing has been smoothed as in Eq. (3.4), and the second particlc feels a different focusing force characterized by the parameter $\Delta k$. This might be due to a difference in energy from the front to the back $c$ the bunch; in this case,

$$
\Delta k=-6 k
$$

where $\delta=\left(E_{2}-E_{1}\right) / E_{1}$. More precisely, for a general lattice we need to evaluate an average chromaticity $\xi$ defined by

$$
\frac{\Delta k}{k}=\xi \frac{E_{2}-E_{1}}{E}=\xi \delta
$$

For typical lattices $\xi$ is close to -1 , and thus the smooth approximation is not too bad.

It is also possible to vary the focusing function along the bunch by the use of RF focusing. This decouples the focusing field from the energy spread but couples it to position within the bunch.

Now let us consider the solution of Eq. (5.1) in which both particles have the same initial offset, $\hat{x}$. For small $\Delta k / k$, the solution for the difference between the transverse positions is given by

$$
x_{2}(s)-I_{1}(s)=\dot{x}\left(2-\frac{e^{2} N W}{2 E k \Delta k}\right) i \sin \left(\frac{\Delta k s}{2}\right) e^{i(k+\Delta k / 2) s} .
$$

To study Eq. (5.4) it is useful to consider three different cases:

\section{Case 1. $\Delta k=0$}

In this case the difference grows linearly

$$
x_{2}(s)-x_{1}(s)=-i \frac{e^{2} N W \hat{z} s}{4 E k} e^{i k s}
$$

which yields an amplification factor given by

$$
\frac{x_{2}-x_{1}}{\hat{x}}=\frac{e^{2} N W s}{4 E k} .
$$

The linear growth is simply due to a linear oscillation driven on resonance. In an actual beam; the growth of the tail of the beam is much faster and has been calculated in Ref. 18.

Case 2. $\Delta k \neq 0, \Delta k$ very small

In this case the linear growth is turned over, leading to a maximum amplification factor of

$$
\frac{x_{2}-x_{1}}{\hat{x}}=\left(2-\frac{e^{2} N W}{2 E k \Delta k}\right) \simeq-\frac{e^{2} N W}{2 E k \Delta k}
$$

the growth stops at $s=\pi / \Delta k$, and there is beating with the maximum amplitude given in Eq. (5.7). 


\section{Case 3. "BNS Damping" ${ }^{19}$}

In this case, if we examine Eq. (5.7), we see that the amplification can be set to zero provided that

$$
\frac{\epsilon^{2} N W}{4 E k \Delta k}=1
$$

This yields no growth at all; in fact simulations of actual beam distributions show genuine damping of the oscillation. ${ }^{19,8}$ This effect, in the past, was referred to as Landau damping; however, Landau damping refers to the lack of growth of coherent oscillations when there is some uncorrelated spread in the oscillation frequencies of the particles in the bunch. In BNS damping, a correlated focusing spread is used to compensate the wake forces when the bunch is rigidly offset to one side. In this case, since both particles are ofiset to one side, the lack of growth is simply due to a cancellation of forces. The wakefield force is exactly cancelled by the additional focusing force for a trailing particle of slightly lower momentum. It is useful to rewrite the condition for the case of momentum spread:

$$
\frac{e^{2} N W \beta^{2}}{8 E \delta_{B N S}}=1
$$

In this case $\delta_{B N S}$ is the half spread in energy required for BNS damping and the average beta function $\beta$ has been used rather than the wave number $k$. Notice that if $\beta \propto E^{1 / 2}$, then $\delta_{\mathrm{BNS}}$ is independent of $E$.

It is useful to see a simulation of the effects of BNS damping. In Fig. 20, you see phase space together with $(x, z)$ space for cases with or without BNS damping. ${ }^{20}$ With no BNS damping, the tail of the bunch grows and phase space is diluted. However, with BNS damping, the beam tail does not grow, and there is little phase space dilution.

\section{B. BNS Damping at the $S L C^{21,22}$}

Recently, we have performed an experiment on the linac at SLAC to test the effects of BNS damping. ${ }^{21,22}$ In the case of the SLC, several sectors were phased so as to enhance the energy correlation from head to tail within the bunch. The following sectors were then phased so as to take out the induced correlation over the rest of the linac. This yielded the energy spread profile as shown in Fig. 21. The peak energy spread occurs at about $1 / 4$ of the way down the linac and is about $2 \%$.

To test the effects of BNS damping, the beam was kicked by a corrector to induce a coherent betatton oscjllation down the linac. The tail growth was measured on a profile monitor. In Fig. 22 you see the size of the tail as a function of the corrector strength with and without B.rS damping. The difference in the slopes of these two cases is about a factor of 10 . This means that the beam is a factor of ten less sensitive to initial offsets due to kicker jitter, power supply jitter, etc. 


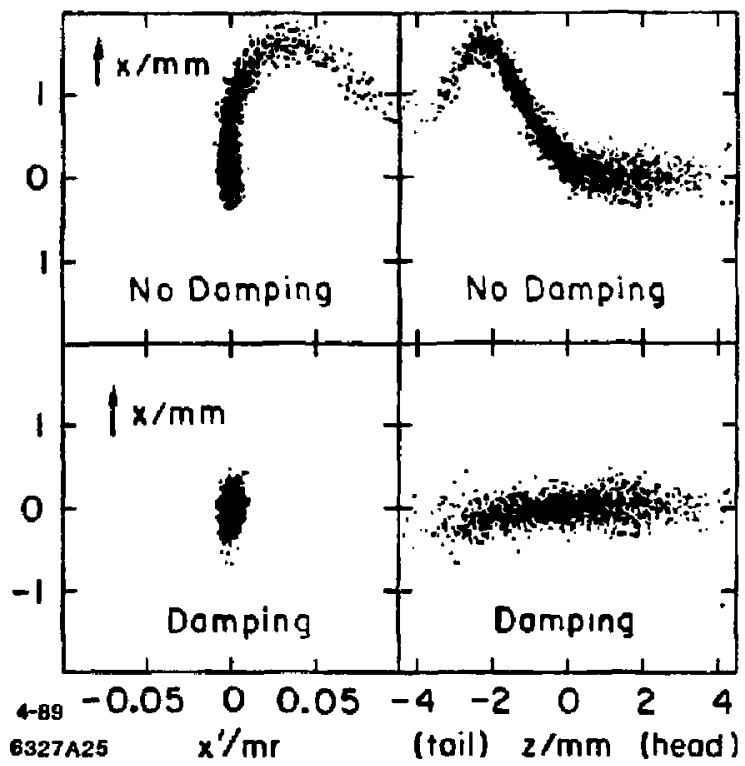

Fig. 20. Calculation of effects of BNS damping at the SLC.

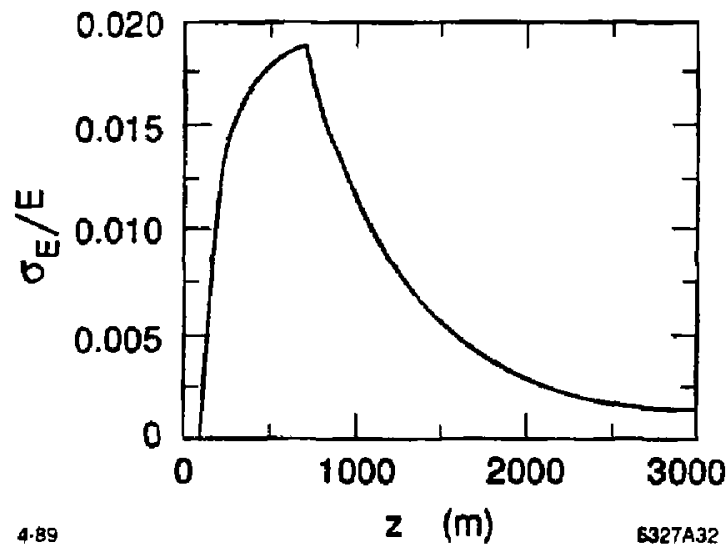

Fig. 21. Relative energy spread us. distance along the linac for BNS damping at SLC.

Qualitatively, the beam seems much more stable with BNS damping than without. Since the experiment, BNS damping is used routinely in the SLC linac to control tail growth. 


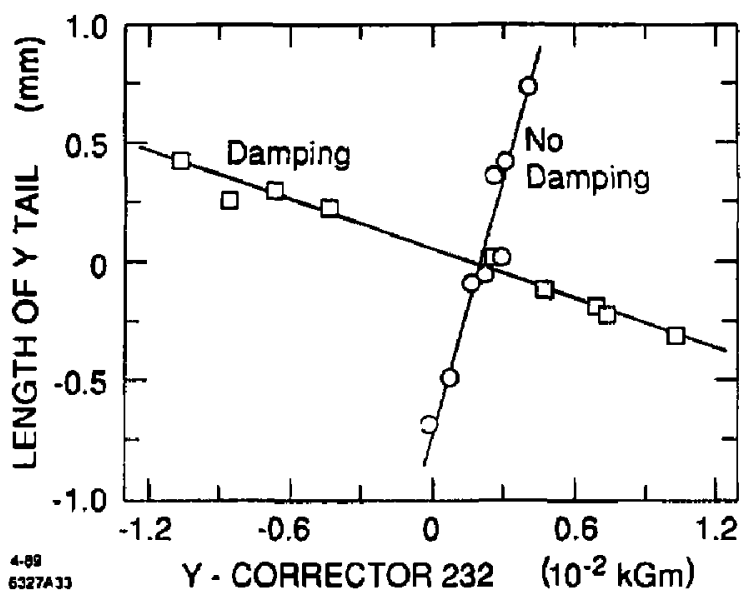

Fig. 22. Effect of a dipole corrector at the beginning of the SLC linac with and without BNS damping.

\subsubsection{Circular Accelerators}

\section{A. Mode Coupling}

The circulation in longitudinal phase space in a circular accelerator causes particles in the front of the bunch to exchange places with the head. In this case, we do not get the simple tail growth as in a linac. Rather, the growth is stabilized at low current. However, the coupling of the head and tail can lezd to an instability via mode coupling. At low current, a bunched beam can oscillate transversely with a coherent tune $\nu_{c}=\nu_{z}+m \nu_{0}$. At higher current these modes move and, if they come close together, they may couple to cause instability.

To illustrate the physics, consider a two-particle model. As the two macroparticles zove longitudinally, the distance between them varies, causing the wakefield to vary. Let us first replace the wakefield by an average value. Then in the two-particle model we have

$$
\left.\begin{array}{c}
x_{1}^{\prime \prime}+\frac{\nu^{2}}{R^{2}} x_{1}=0 \\
x_{2}^{\prime \prime}+\frac{\nu^{2}}{R^{2}} x_{2}=\frac{N e^{2} \bar{W}_{\perp}}{2 E}
\end{array}\right\} 0<t<t_{s} / 2,
$$

and so on. The basic parameter is the relative growth of a tail particle in one half of a synchrotron oscillation,

$$
\eta=\frac{e^{2} N \bar{W}_{\perp} R_{c t}}{8 E \nu}
$$


For $\eta$ small, the net effect of the circulation is a slight shift in frequency of the two modes. The first mode (mode 0 ) in which the particles move in phase has its frequency shifted down while the second mode (mode -1), in which the particles move out of phase, has its frequency shifted up. When these two modes collide $(|\eta|=2)$ there is an instability.

The actual bunch is not composed of two macroparticles. In practice, there are many morc modes to consider. To do this, one uses the Vlasov equation to calculate how all the various modes couple. In Fig. 23, you see a calculation of mode couplirg for a short bunch in a ring with a broad band impedance. ${ }^{23,24}$ You see that it is modes 0 and -1 which ultimately couple to produce the instability. In the case of more complicated impedances and/ $\omega_{i}$ longer bunches, the higher modes become important. In this case, it is possible for the beam to remain stable even after modes 0 and -1 pass through each other.

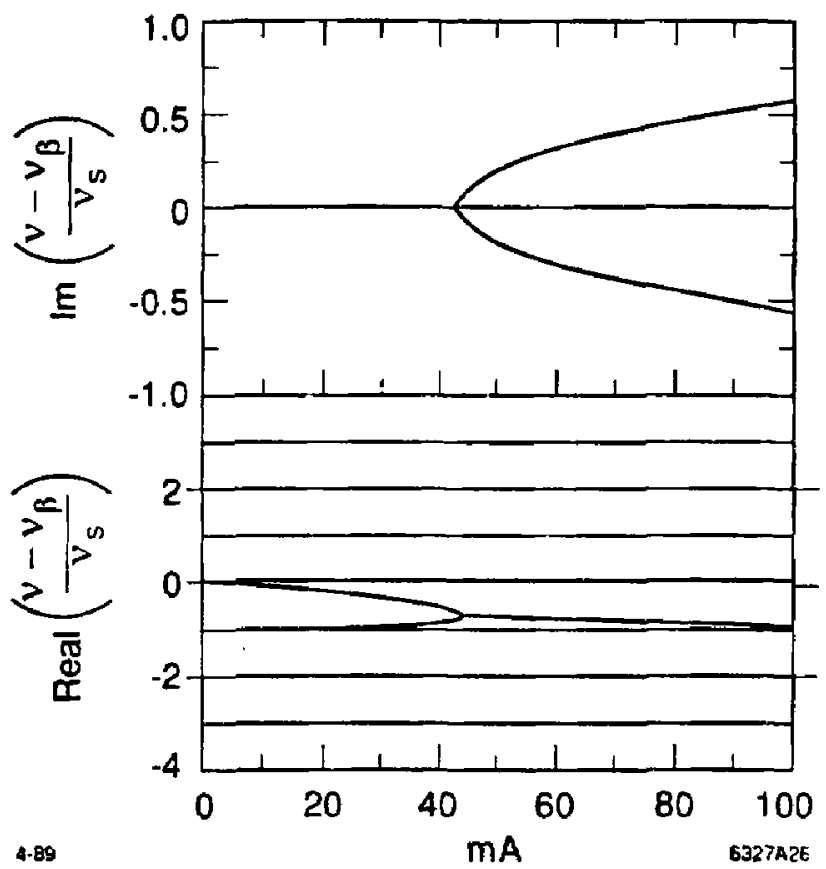

Fig. 23. Mode cou pling with a broad band impedance model for the SLC damping ring.

There have been many experiments on the mode coupling instability. At PEP an experiment was performed to test the effect of feedback on the mode coupling instability. ${ }^{25}$ There had been calculations to indicate that reactive feedback (shifting the coherent tune) could increase the threshold by a factor of 2 or more. ${ }^{26,27,24}$ In Figs. 24 and 25 , you see the characteristics of the instability. Fig. 24 shows the accumulntion of current in PEP abruptly change due to a 
beam loss. Fig. 25 shows the tune shift of mode 0 as the current in the bunch is varied. I $\mathrm{I}_{\mathrm{i}}$ the experiment, two types of feedback were attempted, "reactive feedback" and "resistive feedback." In the first case, the frequency of mode 0 is shifted up to cause the modes to collide later; in the second case, the dipole mode is very strongly damped. Both types of feedback proved to be experimentally successful and improved the threshold by more than a factor of 2.

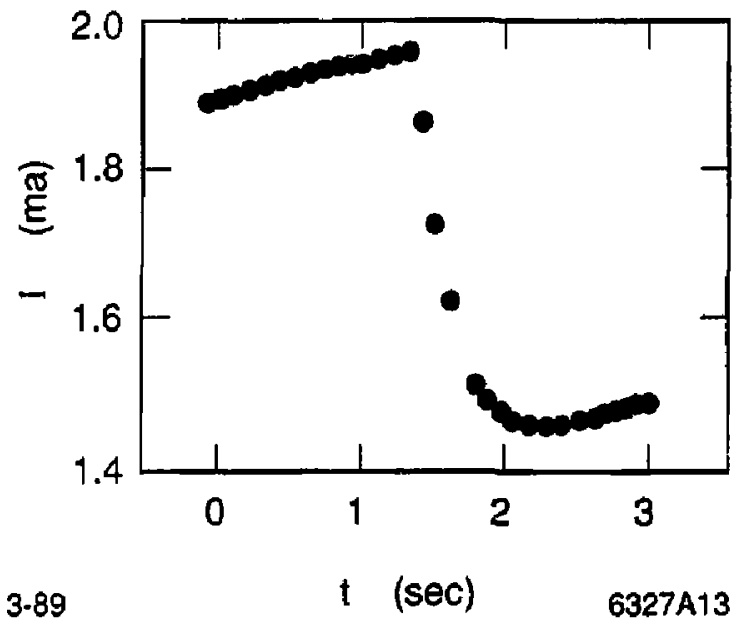

Fig. 24. Current vs, time during injection in PEP. The loss is the mode coupling instability.

\section{B. The Head-Tail Effect 28}

In the previous section we assumed a zero chromaticity which led to the cancellation of the coherent growth of the tail due to the circulation in longitudinal phase space. If the chromaticity is not zero, this cancellation is not perfect, and we can have instability or damping even for small current. The key difference is that the betatron frequency is then a function of $\epsilon$ via

$$
\omega_{\beta}(\epsilon)=\omega_{\beta}^{0}+\omega_{0} \xi \epsilon
$$

where $\xi$ is the chromaticity. If we integrate Eq. (5.12), we find the betatron phase,

$$
\phi_{\beta}(t)=\omega_{\beta}^{0} t-\xi \frac{\omega_{0}}{\alpha} \tau(t)
$$

Thus the phase lags or leads depending upon the sign of $\xi$ and $\tau$. This causes a coherent growth rate or coherent damping rate $\propto \xi$. For modes $\omega_{\beta}^{k} \simeq \omega_{\beta}^{0} n+$ mws we find that $\xi>0$ corresponds to stability for $m=0$. This is a primary reason that the chromaticity must be corrected and slightly positive in a storage ring. 


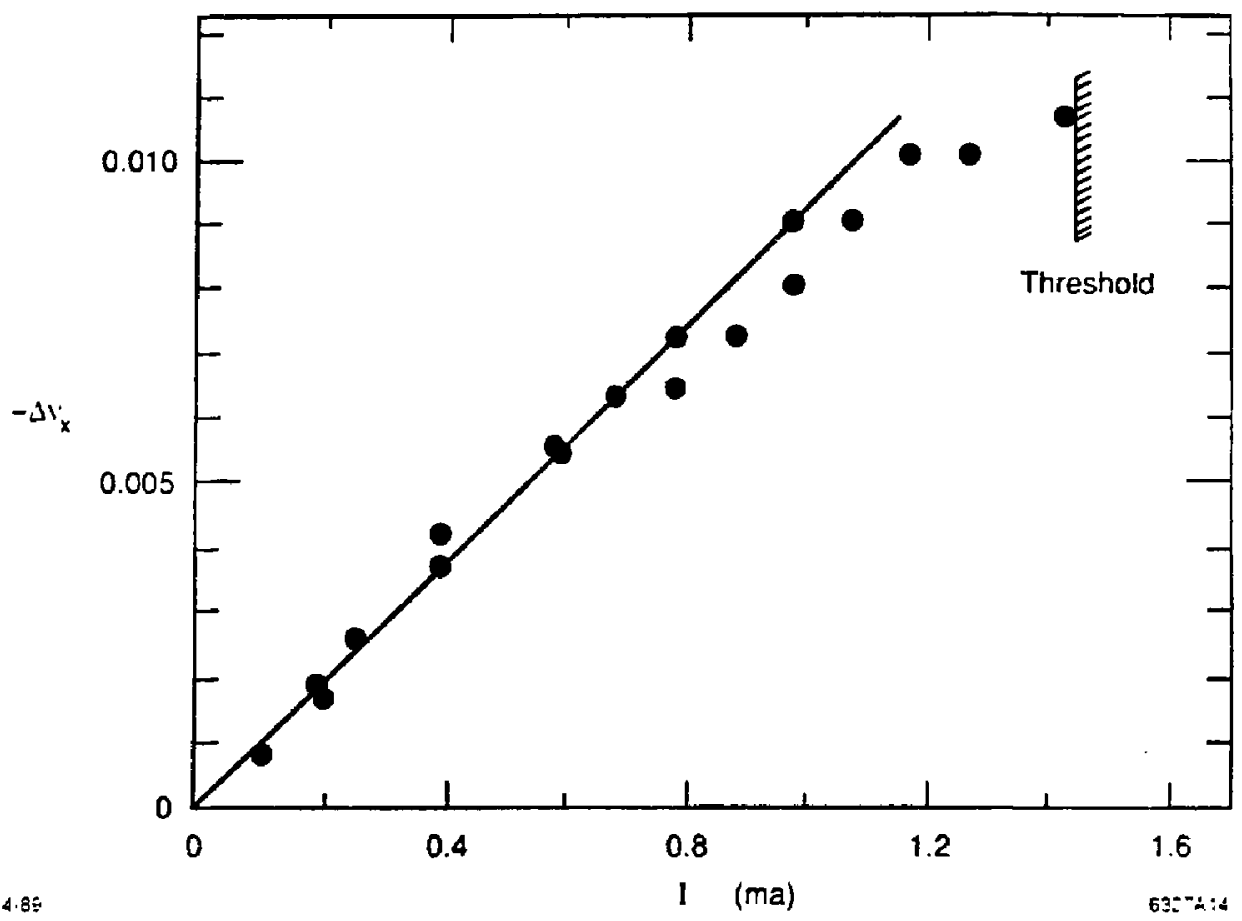

Fig. 25. Coherent shift of horizontal tune vs, current in PEP at $4.5 \mathrm{GeV} .^{25}$

To calculate the effects of the chromaticity on the other modes, it is necessary to solve the Vlasov equation. One could use the two particle model as in the previous section; however, this gives estimates which are not accurate for the higher modes.

In Fig. 26 you see a calculation of the frequency shifts for many coupled low-order modes with non-zero positive chromaticity. ${ }^{23,24}$ The real frequency shifts are similar to those calculated with $\xi=0$; however, now we have coherent damping of mode $m=0$ and growth of effectively; all other modes, with mode $m=1$ having the largest growth rate. If we continue out past the threshold for mode coupling, in $F$ ig. 26, we see that modes 0 and -1 interact once again nea: the threshold of Fig. 23 to induce a larger growth rate for the unstable mode.

In practice there are other damping mechanisms present which can stabilize the higher modes. These are Landau damping due to nonlinear spreads in the betatron frequency and radiation damping in electron storage rings. Therefore, we usually observe beam stability until the mode coupling instability increases the growth rate sufficiently.

The head-tail effect has been observed in many storage rings and accelerators. In Ref. 29 the head-tail effect was studied in SPEAR. In particular, they showed that with positive chromaticity the head-tail effect yields coherent damping of the bunch without dilution of phase space. By 

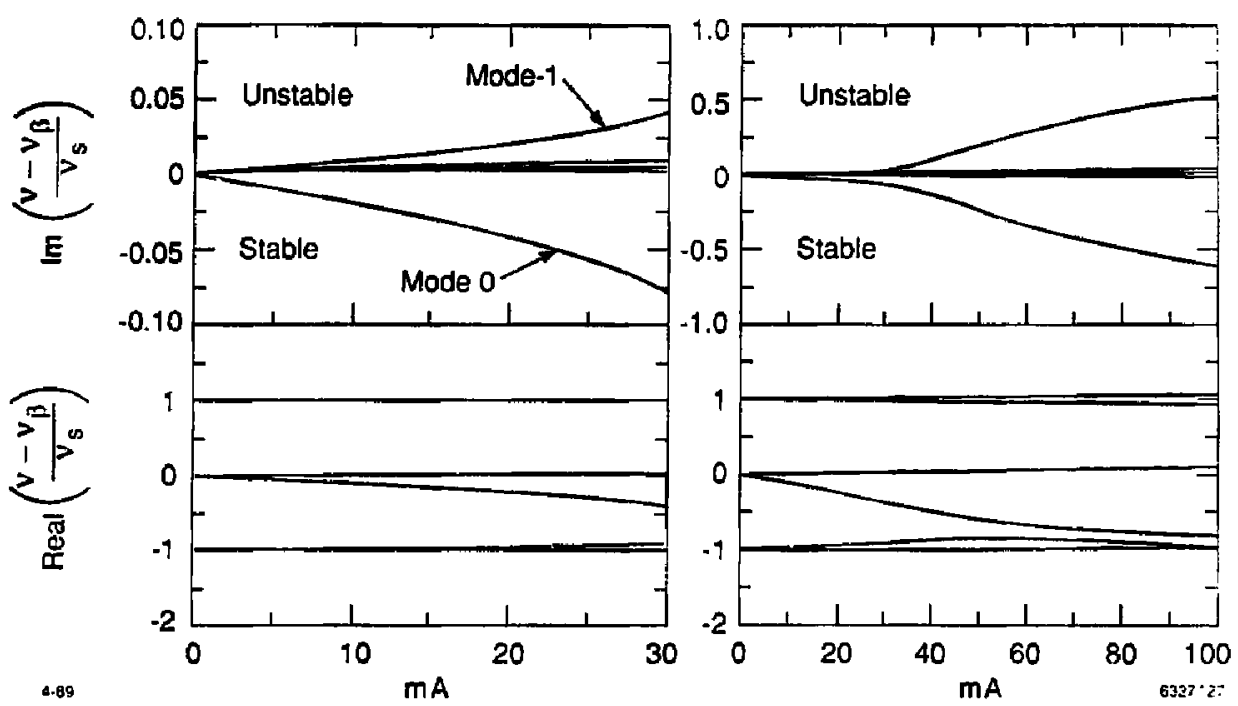

Fig. 26. The head-tail effect and mode coupling with positive chromaticity.

using octupoles, they produced a nonlinear tune spread large enough to provide Landau damping which overwhelmed the head-tail effect. In this case they observed that phase space was diluted as one would expect.

\section{Fast Blow-up of Single Bunches ${ }^{30,23}$}

We have seen that mode coupling can lead to instability for short bunches. What happens if the bunches get very long and the synchrotron frequency is very low? In this case, it is possible that mode coupling will not occur, at least not in the way in which it was described earlier. For long bunches the modes 0 and -1 couple only weakly, and thus there is sometimes no instability induced by their crossing. In addition, if the spread in betatron frequencies gets to be large compared to the synchrotron frequency, then the mode coupling instability may be Lardau damped.

However, this does not mean that there is no instability; it means that it is useful to use a different analysis method. In this case it is useful to use coasting beam theory as we did in Section 4.2.1 ${ }^{32}$ for the longitudinal. In fact it häs been shown that under conditions similar to those stated in Section 4.2.1, there is a transverse coasting-beam-like instability in a bunched beam. $^{30,23}$ Once again for a fast instability with a short wakefield the center of the bunch cannot tell that it is not part of a coasting beam with current equal to the peak current. In this case there is a threshold given by

$$
\frac{e I_{p}\left|Z_{\perp}\left(\omega_{c}\right)\right| \vec{\beta}}{4 \sqrt{2 \pi} E \alpha \sigma_{\epsilon} n_{\epsilon}} \leq 1
$$

where $I_{p}$ is the peak current, $\bar{\beta}$ is the average beta function and $n_{c}=\omega_{c} / w_{0}$ is the mode number 
at the impedance peak. In Eq. (5.14) the chromaticity has been set to zero as it has little effect on this threshold.

\subsection{MULTJ-BUNCH}

\subsubsection{Linear Accelerators}

\section{A. Cumulative Beam Break-up 31,32}

In a train of bunches in a linac, the transverse wake force acting on a bunch due to all preceding bunches causes it to be deflected off axis. As in the two-particle model, if all bunches have very nearly the same transverse focusing strength, the effect is resonant. The first bunch drives the second yielding linear growth in $s$. The first and second drive the third, yielding linear plus quadratic growth in $s$, and so forth.

The equation of motion for the nth bunch is given by

$$
\gamma x_{n}^{\prime \prime}+\gamma^{\prime} x_{n}^{\prime}+\gamma(s) k_{n}^{2}(s) x_{n}=\frac{N e^{2}}{m c^{2}} \sum_{j=1}^{n-1} W_{\perp}[(n-j) \ell] x_{j}(s)
$$

where $\gamma$, the relativistic factor, changes due to acceleration, $k_{n}(s)$ is the smoothed focusing of the $n$th bunch, and $\ell$ is the bunch separation.

The first observation of cumulative beam break-up at SLAC occurred April 27, $1966 .{ }^{33}$ In this case there were about 4,500 bunches each with $5 \times 10^{7}$ electrons spaced $10.5 \mathrm{~cm}$ apart. This yields a 1.6- $\mu$ sec pulse of electrons with $25-\mathrm{mA}$ current.

In contrast, the parameters for SLC are quite different. SLC accelerates 3 bunches $\left(e^{+}, e^{-}, e^{-}\right)$ with currents in the range $1-5 \times 10^{10} /$ bunch but with about 60 -nsec spacing. There are no multjbunch problems for SLC due to the large burch spacing.

For next generation linear colliders to achieve high luminosity, it will be necessary to extract as much energy as possible from the RF. This eads to trains of bunches of 10 to 20 with about I-2 $\times 10^{10}$ particles per bunch. In this case since the bunches are rather close and the $R F$ frequency is quite high, cumulative beam break-up can be a serious problem. Consider the example shown in Fig. 27, In this case the amplitude of the last bunch in a train of 10 has grown by a factor of $10^{5}$ by the end of the linac; this is clearly an unacceptable situation. In the next section we examine some cures for this problem.

B. Cures for Beam Break-up $34,35,36$

There are several possible cures for beam break-up.

1. We could reduce the wakefield couplinz one bunch to the next. This could be done by decreasing the RF frequency, or, at fix.d RF frequency, by increasing the bunch spacing 


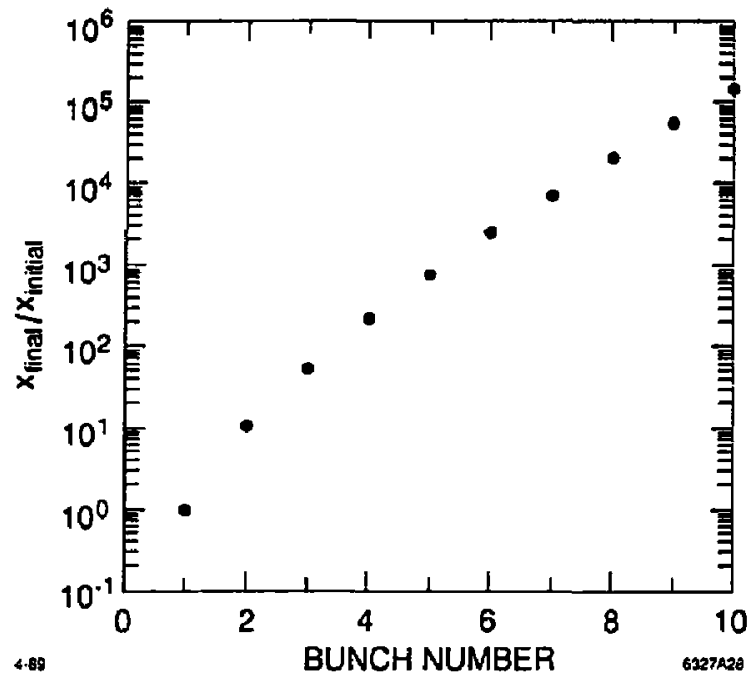

Fig. 27. Growth due to cumulative beam break-up in an undamped $17-\mathrm{GHz}$ traveling wave structure.

to allow the natural damping of the wakefield to reduce it. Finally, one could design the structure in such a way that the bunches fall close to the zero crossings of the wakefield.

2. We could decrease the number of bunches which are coupled together via the wakefield. As you see from Fig. 27, the earlier bunches are disturbed much less than the later ones in a short train of bunches. To accomplish this, we could simply reduce the number of bunches. This would be self defeating because we would like a large number to obtain large luminosity. $A$ better method is to damp the wakefield by some means so that only a few bunches are coupled. Ideally one could imagine damping the wakefield between bunches to effectively decouple the train. If damping is difficult, it may be sufficient to introduce a spread in frequencies in the wakefield by changing the cavity design from cell to ceil. This yields an effective damping of the wake due to the decoherence of the modes from different cavities. The spread in frequencies in the SLAC linac due to the constant gradient taper of the accelerating sections has helped greatly with cumulative beam break-up at SLAC. In the SLC mode, this makes bunch spacings of 10-25 nsec possible for currents of 2-5 $\times 10^{10}$.

3. Finally, we could change the focusing from bunch to bunch to move off resonance. This is suggested by the success of BNS damping in a single bunch. Unfortunately, the required frequency spreads are large and difficult to achieve for a long train of bunches.

For a next generation linear collider at high frequency, extreme measures are necessary. In Table 1, you see the parameters for a calculation of a next generation linear collider. In this case, two cures were selected; damping the transverse wake combined with tuning the frequency of the lowest zominant mode to cause the bunches to fall close to zero crossings of the wake. The results 
are shown in Fig. 28, which plots the maximum blow-up of any bunch in the train versus the tuning of the frequency of the lowest dipole mode for several different values of $Q .{ }^{35}$ We see that it is indeed possible to cure the instability using these techniques provided that $Q$ 's from 15 to 50 can be obtained together with the desired tuning of the transverse mode.

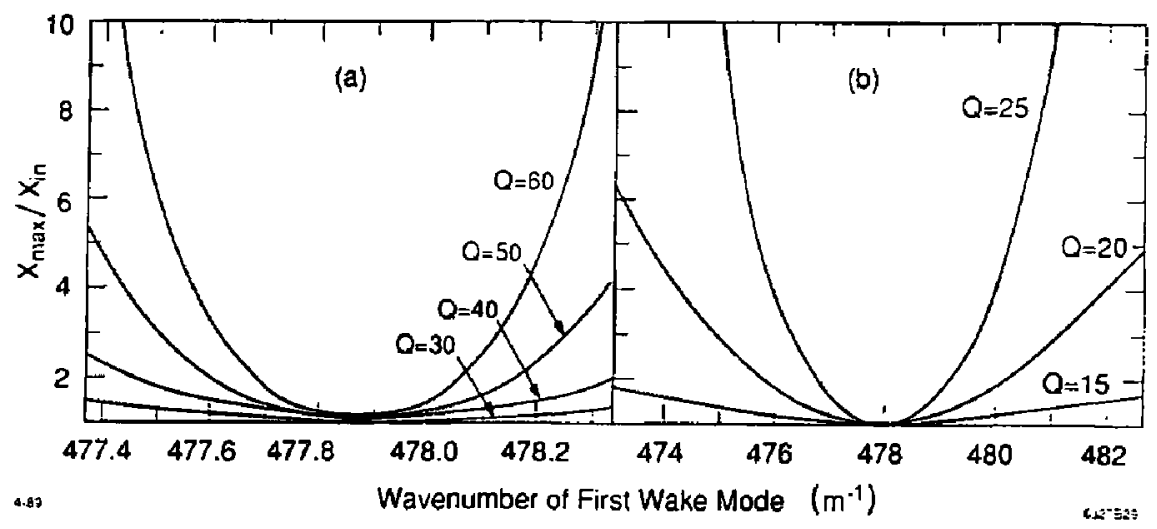

Fig. 28. Maximum growth of any bunch in the train vs. tuning of the first dipole made of the traveling wavt structure

\begin{tabular}{|c|c|}
\hline \multicolumn{2}{|c|}{ Table 1: Parameters for Main Linacs at $17.1 \mathrm{GHz}$} \\
\hline Number of bunches & 10 \\
\hline Number of particles per bunch & $1.67 \times 10^{10}$ \\
\hline Bunch spacing $\ell$ & $21.0 \mathrm{~cm}$ \\
\hline Initial energy of linac & $18 \mathrm{GeV}$ \\
\hline Final energy of linac & $500 \mathrm{GeV}$ \\
\hline Linac length & $3000 \mathrm{~m}$ \\
\hline Initial beta function & $3.2 \mathrm{~m}$ \\
& $\left(k_{0}=0.3125 \mathrm{~m}^{-1}\right)$ \\
\hline
\end{tabular}

One method which has been proposed to damp transverse and higher longitudinal modes is to use slots in the irises coupled to radial waveguides. ${ }^{39}$ In experimental tests $Q$ 's as low as 20 have been measured. In Fig. 29 you see an artist's conception of such a structure. Clearly, damped structures could have applications in storage rings and circular accelerators as well as linacs. 


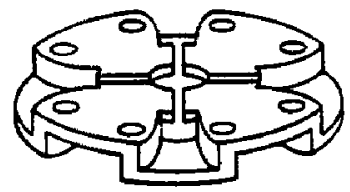

(a)

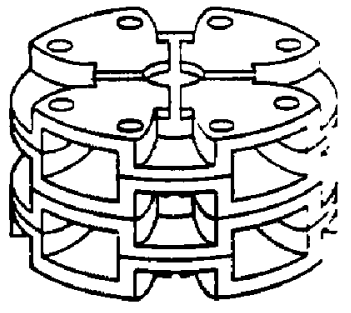

(b) 652:436

Fig. 29. Structure with slots coupled to radial waveguides designed to damp transverse and higher-order longitudinal modes. ${ }^{39}$

\subsubsection{Circular Accelerators}

\section{A. Coupled Batches of Bunches 37,38}

For a circular accelerator the coupled bunch problem is somewhat more complex than in the linac because the wakefield from a bunch can interact with that bunch on subsequent turns. However, for equally spaced bunches of equal intensity, there is a standard theory for coupled bunch motion similar to that for the longitudinal. ${ }^{10}$ In the case of unequal spacing and/or damped wakefields other techniques are useful.

To be specific, let us consider a storage ring filled with several batches of about 10 closely spaced bunches. Let us first ignore the other batches and consider the stability of a single batch. The equation of motion for the position of the rigid motion of the bunch is

$$
x_{n}^{\prime \prime}+2 \alpha x_{n}^{\prime}+k^{2} x_{n}=\frac{N e^{2}}{E} \sum_{j=1}^{n-1} W[(n-j) \ell] x_{j}+\frac{N e^{2}}{E} \sum_{j=1}^{n \text { Tot }} \sum_{q=1}^{\infty} W[(n-j) \ell+q C] x_{j}
$$

The last term comes from the wakefield of all preceding turns. Let us consider the case in which we damp the wakefield in less than one turn but not within the bunch train. In this case the batch of bunches does not see its wake on preceding turns. We refer to this as the intermediate wakefield regime. Notice that in this model the focusing function has been replaced by a smooth approximation, and the wake function is uniform around the ring. Actually, the wakefield is localized to the RF structures and other high- $Q$ objects. For a more accurate representation, one can introduce a sequence of maps which describe the dynamics. These two methods yield identical results provided that the coherent tune shift $\Delta \nu_{c} \leqslant 1 / 2 \pi$, and provided the tune is far from integers.

Note that we have included a coherent damping factor in the differential equation. This factor is due to one of three causes:

1. First, in an electron ring the motion of each particle is coherently damped due to radiation. 
2. If there is a betatron tune spread due to nonlinearities, then the coherent motion will bc damped by phase dilution. This Landau damping is, in general, not exponential.

3. As we saw from the section on the head-tail effect, for positive chromaticity the simple dipole motion of the bunch is damped coherently due to the interaction of the short wakefield and the longitudinal circulation within the bunch.

Without specifying the mechanism, we include this damping factor explicitly in the model.

With all the conditions above, the storage ring acts like a very long linac with a cavity now and then; however, the key difference is the damping of the betatron oscillation which does not occur in a linac. In the case of a linac, the bunches at the tail of a short train can grow substantially as we saw in Section 5.2.1. However, for a storage ring, due to the exponential damping, the bunch train will ultirnately be stable. In spite of this, the non-exponential growth of tail bunches can lead to very large transient instability.

Consider los example the case shown in Fig. $30 .^{38}$ You see that the first bunch damps exponentially as expected. However, later bunches show a transient growth which can get quite large and can persist for several damping times.

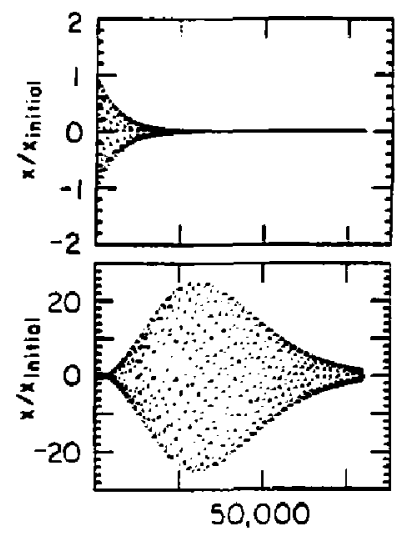

6327as1 TURN NUMEER

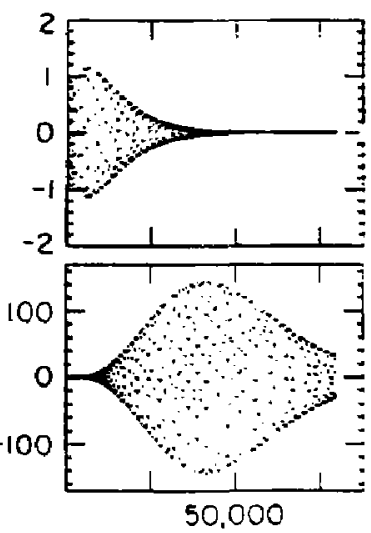

TURN NUMEER 4-E'

Fig. 30. Transient growth in a batch of bunches in a storage ring.

This type of transient instability has not been appreciated until recently. However, it is obvious when viewed in the time domain. If we do the normal frequency domain calculation as in Section 4.2.2 for the longitudinal case, we would calculate all the modes (20 in the case of Fig. $30)$, and they all would have an imaginary part less than zero. ${ }^{37,38}$ That is, all 20 modes would be deemed stable. However, to treat the transient behavior properly, we must use the Laplace transform and go to the time domain. In this case, in spite of the damping of all 20 modes, the transient behavior which is recovered is exactly that shown in Fig. 30. 
This type of instability can lead to particle loss even though the beam is asymptotically stable. It is also important for damping rings where the beam is extracted a tather short time after injection.

\section{CONCLUDING REMARKS AND ACKNOWLEDGEMENTS}

This paper has presented an overview of collective effects in both circular and linear accelerators. A complete overview of this subject would easily fill one or two books and a complete list of references would be longer than this entire paper. The compromise which was made was to touch on a few subjects in each category, giving some theory, some physical discussion, and some experimental results where possible. The interested reader who wishes details on any of the subtopics discussed here should pursue the study beginning with the review articles in Ref. 1-3.

I would like to thank Kathy Asher for help in preparing the manuscript, Karl Bane and Kathy Thompson for useful discussions and for help with figure preparation, and finally Juinn Ming Wang, Robert Warnock, and Perry Wilson for continuing discussions of collective effects.

\section{REFERENCES}

1. Theoretical Aspects of the Behavior of Beams in Accelerators and Storage Rings, CERN 77-13.

2. Physics of High Energy Particle Accelerators, Proc. of the U.S. Particle Accelerator Summer Schools 1981-1985, AIP Conf. Proc. No. 87, 105, 127, 153.

3. CERN Accelerator School General Accelerator Physics, CERN 85-19.

4. J. Haissinski, Il Nuovo Cimento 18b, No. 1, 72 (1973).

5. K. L. F. Bane and R. D. Ruth, IEEE Particle Accelerator Conf. (1989) (in press) and SLAC-PUB-4905.

6. L. Rivkin et al., in Proc. of European Particle Accelerator Conf., Rome, Italy (1988) (in press) and SLAC-PUB-4645.

7. K. L. F. Bane, in Proc. of European Particle Accelerator Conf., Rome, Italy (1988) (in press) and SLAC-PUB-4618.

8. K. L. F. Bare, in Physics of High Energy Part. Acc., AIP Conf. Proc. 153, Ed. M. Month and M. Dienes, 971 (1987).

9. L. J. Laslett, BNL-7534 (1963).

10. R. D. Ruth, in Proc. of ICFA/INFN Workshop on Physics of Linear Colliders, Capri, Italy, 1988, and in SLAC-PUB-4541.

11. R. Palmer, private communication.

12. A. K. Neil and A. M. Sessler, Rev. Sci. Instr. 36, 429 (1965).

13. L. D. Landau, J. Physics USSR 10, 25 (1946).

14. D. Boussard, CERN LABII/RF/INT/75-2 (1975).

15. J. M. Wang and C. Pellegrini, Proc. of the 11th Int. Conf. on High Energy Acc., Geneva, Switzerland, p. 554 (1980). 
16. T. Suzuki, Y. Chin and K. Satoh, Particle Accelerators 13, 179 (1983).

17. K. Robinson, CEAL Report TM-183 (1969).

18. A. Chao, B. Richter, and C. Yao, Nucl. Instr. and Meth. 178, 1 (1980).

19. V. Balakin, A. Novokhatsky, V. Smirnov, Proc. of the 12th Int. Conf. on High Energy' Accelerators, FNAL, Batavia, IL, 199 (1983).

20. K. L. F. Bane, SLAC AP/69 (1988).

21. J. Seeman et al., submitted for publication, SLAC-PUB-4968.

22. J. Seeman et al., IEEE Part. Accel. Conference, Chicago, IL, 1989, SLAC-PUB-4887.

23. R. D. Ruth, PhD Dissertation, BNL 51425.

24. R. D. Ruth, Proc. 12th Int. Conf. on High Energy Accelerators, FNAL, Batavia, IL, 389 (1983).

25. M. Donald et al., LEP Note 553 (1986).

26. R. D. Ruth, CERN-LEP-TH/83-22 (1983).

27. S. Myers, LEP Note 436 (1983).

28. C. Pellegrini, Il Nuovo Cimento 64A, No. 2, 447 (1969).

29. The Spear Group, IXth Int. Conf. on High Energy Acc. (1974), p. 338.

30. R. D. Ruth and J. M. Wang, IEEE Trans. Nucl. Sci. NS-28, No. 3, 2405 (1981).

31. W. K. H. Panofsky and M. Bander, Review Sci. Instr. 39, No. 2, 206 (1968).

32. R. H. Helm and G. A. Loew, in Linear Accelerators, ed. P. M. Lapostolle, A. L. Septier, North-Holland Pub. Co., 173 (1970).

33. R. Helm, G. Loew, W. K. H. Panofsky, The SLAC Blue Book, W. A. Benjamin, Inc., New York, 1968 p. 88.

34. K. A. Thompson and R. D. Ruth, in Proc. of IEEE Part. Acc. Conf., Chicago, IL (1989) (in press) and SLAC-PUB-4873.

35. K. A. Thompson and R. D. Ruth, in Proc. of Snowmass 88, SLAC-334 and SLAC-PLB4800.

36. K. A. Thompson and R. D. Ruth, submitted for publication and SLAC-PUB-4801.

37. K. A. Thompson and R. D. Ruth, in Proc. of IEEE Part. Acc. Conf., Chicago, IL (1989) (in press) and SLAC-PUB-4872.

38. K. A. Thompson and R. D. Ruth, Submitted for publication and SLAC-PUB-4962.

39. R. B. Palmer, in Proc. of Snowmass 88, SLAC-334, and SLAC-PUB-4542 (1988).

40. E. D. Courant and A. M. Sessler, Rev. Sci. Instr. 37, No. 11, 1579 (1966).

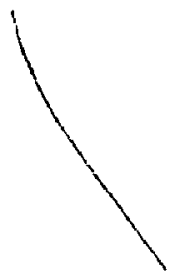

